AEI-2009-066

\title{
Analytic solution of the multiloop Baxter equation
}

\author{
M. Beccaria ${ }^{a}$, A.V. Belitsky ${ }^{b}$, A.V. Kotikov ${ }^{c}$ And S. Zieme ${ }^{d, e}$ \\ ${ }^{a}$ Physics Department, Salento University and INFN, \\ 73100 Lecce, Italy \\ ${ }^{b}$ Department of Physics, Arizona State University \\ Tempe, AZ 85287-1504, USA \\ ${ }^{c}$ Bogoliubov Laboratory of Theoretical Physics \\ Joint Institute for Nuclear Research \\ 141980 Dubna, Russia \\ ${ }^{d}$ Max-Planck-Institut für Gravitationsphysik \\ Albert-Einstein-Institut \\ Am Mühlenberg 1, D-14476 Potsdam, Germany \\ ${ }^{e}$ Faculty of Science, University of Herat, Afghanistan
}

\begin{abstract}
The spectrum of anomalous dimensions of gauge-invariant operators in maximally supersymmetric Yang-Mills theory is believed to be described by a long-range integrable spin chain model. We focus in this study on its $s l(2)$ subsector spanned by the twist-two singletrace Wilson operators, which are shared by all gauge theories, supersymmetric or not. We develop a formalism for the solution of the perturbative multiloop Baxter equation encoding their anomalous dimensions, using Wilson polynomials as basis functions and Mellin transform technique. These considerations yield compact results which allow analytical calculations of multiloop anomalous dimensions bypassing the use of the principle of maximal transcendentality. As an application of our method we analytically confirm the known four-loop result. We also determine the dressing part of the five-loop anomalous dimensions.
\end{abstract}




\section{Introduction}

The success of gauge theories in accurately describing the laws of nature is based on the availability of computational techniques, see e.g., Ref. [1], which allow for a systematic improvement of approximations involved. Perturbative expansions in the gauge coupling constant $g_{\mathrm{YM}}$ are conventionally deduced from Feynman diagrams. However, due to uncontrollable proliferation of the latter at higher orders in $g_{\mathrm{YM}}$, the rules quickly become unmanageable, making direct computations already at four-loop order highly nontrivial and require massive computer manipulations. On top of this, individual Feynman diagrams obscure underlying properties of the theory and reveal simple results enjoying sometimes enhanced symmetries only in their sum. One was therefore compelled to search for an alternative approach which presented itself recently.

On the one hand, some time ago it was established that at weak coupling one-loop spectra of anomalous dimensions of maximal-helicity gauge-invariant operators in QCD coincide with energy spectra of a one-dimensional non-compact Heisenberg magnet [2, 3]. The latter can be diagonalized by means of the traditional Bethe ansatz formalism of integrable systems and yields anomalous dimensions of the corresponding four-dimensional gauge theory. These simplifications are echoed by higher loop contributions, especially in supersymmetric gauge theories. It was found in Refs. [4, 5] that all single-trace operators in planar, maximally supersymmetric gauge theory

$$
\mathcal{O}=\operatorname{tr}\left(X\left(D_{+}^{2} X\right) Y Z X \lambda X F_{+\perp}\left(D_{+} Y\right) \bar{\lambda} \ldots\right)
$$

can be described by a long-range integrable spin-chain model with elementary excitations identified with the particle fields $Y, Z, \lambda$ etc. of the gauge theory and/or covariant derivatives $D_{\mu}$ acting on them propagating on the vacuum state $|0\rangle=\operatorname{tr}\left(X^{L}\right)$. Less supersymmetric Yang-Mills theories entertain integrability only in certain closed subsectors under renormalization group evolution [6].

On the other hand, the AdS/CFT correspondence [7] conjectures that the strongly coupled $\mathcal{N}=4$ Yang-Mills theory is dual to a free type IIB super-string theory on an $\mathrm{AdS}_{5} \times \mathrm{S}^{5}$ background. The latter was found to be classically integrable as well [8]. Using this conjecture as a virtue led to a suggestion of an integrable structure which interpolates between weak and strong coupling regimes. Though the underlying spin chain model is not known, a set of Bethe ansatz equations is nevertheless available [5, 9], which has passed a number of non-trivial tests at weak coupling, see e.g. [9] and [10], as well as at strong coupling by positive comparison with perturbative string theory, see e.g. [11] and [12].

These findings suggest to use the putative integrable structure as an alternative to the conventional Feynman diagrams technique for multiloop calculations of anomalous dimensions. In this paper, we develop a practitioner's formalism building up on earlier considerations based on the all-order Baxter equation [13, 14] for finding the spectrum of twist-two Wilson operators

$$
\mathcal{O}=\operatorname{tr}\left(X D_{+}^{M} X\right)
$$

These arise in all gauge theories albeit with a different field content, the scalar $X$ being specific to supersymmetric cousins of QCD. Their anomalous dimensions have been obtained 
diagrammatically to a considerably high-order [15, 16, 17, 18, 19].

The Baxter equation is advantageous over the Bethe ansatz formalism if one is interested in a systematic analytical framework. However they both enter on equal footings for numerical studies, and Bethe equations were used in the past together with the principle of maximal transcendentality [20] to perform phenomenal computations [21, 22].

Our following consideration is a generalization of the study in Ref. [23] which was based on a deformation of the solution to the one-loop Baxter equation. What will differ in the current work is that we will introduce a new basis of functions used in the construction of next-to-leading order solutions, the so-called Wilson polynomials. For comparison we also present the basis of continuous Hahn polynomials used in [23]. Furthermore we obtain a new form for non-polynomial contributions which is free from multiple sums involving Stirling numbers. The latter property is essential for obtaining analytical results for anomalous dimensions in terms of nested harmonic sums. Our subsequent presentation is organized as follows. In the next section, we briefly review the formalism of the Baxter equation in maximally supersymmetric gauge theory and then present a novel form of the solution in two- to four-loop order in the gauge coupling. The non-polynomial parts of the Baxter equation are analyzed in Section 5 using the Mellin transform technique. We present the analytic form of anomalous dimensions and then in Section 7, we discuss the reciprocity properties including the dressing part of the five-loop anomalous dimensions. Finally, we conclude. Several appendices summarize basic definitions required in the main body of the paper and details of calculations which are two lengthy to be presented in the main text.

\section{Baxter equation}

The spin-chain description allows one to calculate anomalous dimensions of Wilson operators as a function of the 't Hooft coupling constant $g^{2}=g_{\mathrm{YM}}^{2} N_{c} /\left(4 \pi^{2}\right)$. However, the formalism based on the Bethe ansatz equations has the drawback that its predictions fail when the order of the perturbative expansion in $g^{2}$ exceeds the length $L$ of the operator under study [24, 25]. This implies that for twist-two operators (1.2) the onset of wrapping effects occurs starting from four-loops already and the complete anomalous dimension is a sum of two terms

$$
\gamma(g)=\gamma^{\text {(asy) }}(g)+\gamma^{(\text {wrap })}(g) .
$$

The first contribution $\gamma^{\text {(asy) }}$ on the right-hand side is determined by the solution to the asymptotic Bethe ansatz equations and can be written in terms of the Baxter function $Q(u)$ as $[13]$

$$
\gamma^{(\mathrm{asy})}(g)=i g^{2} \int_{-1}^{1} \frac{d t}{\pi} \sqrt{1-t^{2}}\left(\ln \frac{Q\left(+\frac{i}{2}-g t\right)}{Q\left(-\frac{i}{2}-g t\right)}\right)^{\prime} .
$$

\footnotetext{
${ }^{1}$ Please note that we use a different convention for the coupling constant then in [23].
} 
The latter is a degree- $M$ polynomial in the spectral parameter $u$ with zeros determined by the Bethe roots $u_{k}$

$$
Q(u)=\prod_{k=1}^{M}\left(u-u_{k}(g)\right)
$$

It obeys an equation known as the asymptotic Baxter equation

$$
\left(x^{+}\right)^{L} \mathrm{e}^{\sigma_{+}\left(u^{+}\right)-\Theta\left(u^{+}\right)} Q(u+i)+\left(x^{-}\right)^{L} \mathrm{e}^{\sigma_{-}\left(u^{-}\right)-\Theta\left(u^{-}\right)} Q(u-i)=t(u) Q(u) .
$$

Compared to the Baxter equation for the familiar non-compact nearest-neighbor XXX Heisenberg spin chain

$$
\mathfrak{B}\left[Q_{0}\right] \equiv\left(u^{+}\right)^{L} Q_{0}(u+i)+\left(u^{-}\right)^{L} Q_{0}(u-i)-t_{0}(u) Q_{0}(u)=0
$$

with factors $\left(u^{ \pm}\right)^{L} \equiv\left(u \pm \frac{i}{2}\right)^{L}$ accompanying the corresponding Baxter polynomials, Eq. (2.4) possesses highly non-trivial dressing 2 factors reflecting coupling-constant dependent dynamics of the four-dimensional Yang- Mills theory. First, the spectral parameter gets renormalized [26] and reads $x=x[u]=\frac{1}{2}\left(u+\sqrt{u^{2}-g^{2}}\right)$, with the assumed conventional notation $x^{ \pm}=x\left[u^{ \pm}\right]$, and, second, the exponents $\sigma$ and $\Theta$ provide the interpolation between weak and strong-coupling expansions [9] and read [14],

$$
\begin{aligned}
\sigma_{ \pm}(u)=\int_{-1}^{1} \frac{d t}{\pi} \frac{\ln Q\left( \pm \frac{i}{2}-g t\right)}{\sqrt{1-t^{2}}}\left(1-\frac{\sqrt{u^{2}-g^{2}}}{u+g t}\right), \\
\Theta(u)=-8 i \sum_{r=2}^{\infty} \sum_{s=r+1}^{\infty}\left(\frac{g}{2}\right)^{r+s-2} C_{r s}(g) \int_{-1}^{1} \frac{d t}{\pi} \sqrt{1-t^{2}}\left(\ln \frac{Q\left(+\frac{i}{2}-g t\right)}{Q\left(-\frac{i}{2}-g t\right)}\right)^{\prime} \\
\times\left\{\left(-\frac{2}{g}\right)^{s-2} \frac{U_{s-2}(t)}{x^{r-1}}-\left(-\frac{2}{g}\right)^{r-2} \frac{U_{r-2}(t)}{x^{s-1}}\right\},
\end{aligned}
$$

with the expansion coefficients given by

$$
C_{r s}(g)=\sin \left(\frac{\pi}{2}(s-r)\right) \int_{0}^{\infty} d v \frac{J_{r-1}(g v) J_{s-1}(g v)}{v\left(\mathrm{e}^{v}-1\right)} .
$$

Since the Bethe roots acquire dependence on the 't Hooft coupling, the Baxter function can be expanded in a perturbative series $Q(u)=Q_{0}(u)+g^{2} Q_{1}(u)+\ldots$ and each term found explicitly as a solution to Eq. (2.4) as we demonstrate next. Notice that all subleading Baxter functions $Q_{\ell>0}(u)$ are polynomials in the spectral parameter of a degree two units lower than the leading $Q_{0}(u)$. To four-loop order, the dressing functions admit the expansion

$$
\sigma^{ \pm}(u)=g^{2} \frac{i}{u} \gamma_{0}^{ \pm}+g^{4}\left[\frac{i}{u} \gamma_{1}^{ \pm}-\frac{1}{4 u^{2}}\left(\left(\gamma_{0}^{ \pm}\right)^{2}+\alpha^{+}\right)+\frac{i}{4 u^{3}} \gamma_{0}^{ \pm}\right]
$$

\footnotetext{
${ }^{2}$ This nomenclature should not be confused with the dressing factor related to the phase $\Theta(u)$, which we also refer to in later sections.
} 


$$
\begin{aligned}
+ & g^{6}\left[\frac{i}{u} \gamma_{2}^{ \pm}-\frac{1}{u^{2}}\left(\frac{1}{2} \gamma_{1}^{ \pm} \gamma_{0}^{ \pm}+2 \gamma_{0}^{ \pm} \beta^{ \pm}-\frac{1}{4}\left(\alpha^{ \pm}\right)^{2}-\frac{1}{4}\left(\gamma_{0}^{ \pm}\right)^{2} \alpha^{ \pm}+\chi^{ \pm}\right)\right. \\
& \left.+\frac{i}{u^{3}}\left(\frac{1}{4} \gamma_{1}^{ \pm}-\frac{1}{12} \gamma_{0}^{ \pm}\left(\left(\gamma_{0}^{ \pm}\right)^{2}+\frac{3}{2} \alpha^{ \pm}\right)+\beta^{ \pm}\right)-\frac{1}{8 u^{4}}\left(\left(\gamma_{0}^{ \pm}\right)^{2}+\alpha^{ \pm}\right)+\frac{i}{8 u^{5}} \gamma_{0}^{ \pm}\right] \\
+ & \ldots, \\
\Theta(u)= & g^{6} \zeta_{3} \Re \mathrm{e}\left[\frac{1}{2 u^{2}} \gamma_{0}^{+}-\frac{i}{u}\left(\left(\gamma_{0}^{+}\right)^{2}+\alpha^{+}\right)\right]+\ldots,
\end{aligned}
$$

where the expansion coefficients are introduced explicitly in Appendix A.

Yet another unknown in Eq. (2.4) is the transfer matrix, which takes the form

$$
t(u)=\Re \mathrm{e}\left(x^{+}\right)^{L}\left(2+\sum_{k \geq 1} \mathfrak{Q}_{k}(g) \Re \mathrm{e}\left(x^{+}\right)^{-k}\right)-\sum_{k \geq 1} \mathfrak{R}_{k}(g) \Im \mathrm{m}\left(x^{+}\right)^{-k} .
$$

Here the upper limits in the sums can exceed the length of the operator in question and the emerging charges $\mathfrak{Q}_{k>L}$ along with $\mathfrak{R}_{k}$ serve to compensate non-polynomial terms arising in the left-hand side of the finite difference equation (2.4) stemming from the expansion of the renormalized rapidity parameter and dressing factors in Taylor series in the 't Hooft coupling. The charges admit perturbative expansions

$$
\begin{aligned}
& \mathfrak{Q}_{k}=\mathfrak{Q}_{k}^{[0]}+g^{2} \mathfrak{Q}_{k}^{[1]}+g^{4} \mathfrak{Q}_{k}^{[2]}+\ldots, \\
& \mathfrak{R}_{k}=\mathfrak{R}_{k}^{[0]}+g^{2} \mathfrak{R}_{k}^{[1]}+g^{4} \mathfrak{R}_{k}^{[2]}+\ldots .
\end{aligned}
$$

And the only non-trivial contributions for $L=2$ operators up to four-loop order read

$$
\begin{aligned}
& \mathfrak{Q}_{2}^{[0]}=-M(M+1), \\
& \mathfrak{Q}_{2}^{[1]}=-(2 M+1) \Re \mathrm{e}\left[\gamma_{0}^{+}\right], \\
& \mathfrak{Q}_{2}^{[2]}=-(2 M+1) \Re \mathrm{e}\left[\gamma_{1}^{+}\right]-\frac{1}{2} \Re \mathrm{e}\left[3\left(\gamma_{0}^{+}\right)^{2}+\alpha^{+}\right], \\
& \mathfrak{Q}_{2}^{[3]}=-(2 M+1) \Re \mathrm{e}\left[\gamma_{2}^{+}\right]+\frac{1}{2} \Re \mathrm{e}\left[\alpha^{+}\left(\left(\gamma_{0}^{+}\right)^{2}+\alpha^{+}\right)-\gamma_{0}^{+}\left(6 \gamma_{1}^{+}+8 \beta^{+}+\zeta_{3}\right)-4 \chi^{+}\right], \\
& \mathfrak{R}_{1}^{[3]}=-\frac{1}{2} \Re \mathrm{e}\left[\gamma_{0}^{+}\left(\left(\gamma_{0}^{+}\right)^{2}+\alpha^{+}\right)\right], \\
& \mathfrak{R}_{2}^{[3]}=0, \\
& \mathfrak{R}_{3}^{[3]}=\frac{1}{8} \Re \mathrm{e}\left[\gamma_{0}^{+}\right] .
\end{aligned}
$$

Here $\mathfrak{Q}_{2}^{[0]}$ is the eigenvalue of the quadratic Casimir operator of the collinear conformal subgroup in the basis of conformal Wilson operators such that the leading order transfer matrix admits the conventional form for the two site non-compact Heisenberg spin chain,

$$
t_{0}(u)=\left(u^{+}\right)^{2}+\left(u^{-}\right)^{2}+\mathfrak{Q}_{2}^{[0]} .
$$

Finally, the solution to the Baxter equation has to be supplemented with the condition of the vanishing quasi-momentum

$$
i \vartheta=\frac{1}{\pi} \int_{-1}^{1} \frac{d t}{\sqrt{1-t^{2}}} \ln \frac{Q\left(+\frac{i}{2}-g t\right)}{Q\left(-\frac{i}{2}-g t\right)}=0,
$$

in order to pick out only cyclic, physical states. 


\section{Wilson vs. Hahn}

It is known for quite some time that the leading order solution $Q_{0}$ for the non-compact two-site Heisenberg magnet is given by the continuous Hahn polynomials 3 [28],

$$
Q_{0}(u)={ }_{3} F_{2}\left(\begin{array}{c|c}
-M, M+1, \frac{1}{2}+i u \\
1,1
\end{array} \mid 1\right)
$$

Proceeding to higher loops, it was demonstrated in Ref. [23] that subleading contributions $Q_{\ell>0}(u)$ to the Baxter function can be obtained by a deformation of the leading order result (3.1). The equations which these corrections obey remain of second order in finite differences, but acquire inhomogeneous terms depending on lower-order functions. This implies that the structure of all polynomial higher-loop contributions can be immediately understood once the building blocks for the two-loop Baxter function are known. To find the latter it suffices to expand both side of the Baxter equation (2.4) to $\mathcal{O}\left(g^{2}\right)$ and find

$$
\begin{aligned}
{\left[\left(u^{+}\right)^{2}-\frac{1}{2} g^{2}\left(1-i \gamma_{0} u^{+}\right)\right] Q(u+i) } & +\left[\left(u^{-}\right)^{2}-\frac{1}{2} g^{2}\left(1+i \gamma_{0} u^{-}\right)\right] Q(u-i) \\
& =\left[\left(u^{+}\right)^{2}+\left(u^{-}\right)^{2}-g^{2}+\mathfrak{Q}_{2}\right] Q(u),
\end{aligned}
$$

with the quadratic conformal Casimir $\mathfrak{Q}_{2} \simeq-\left(M+1+\frac{1}{2} g^{2} \gamma_{0}\right)\left(M+\frac{1}{2} g^{2} \gamma_{0}\right)$ renormalized by the one-loop anomalous dimension $\gamma_{0}=2 S_{1}(M)$ to this order of perturbation theory. Matching this to the equation obeyed by the continuous Hahn polynomials, we obtain the result for the Baxter function with incorporated two-loop corrections

$$
Q(u)=N(g)_{3} F_{2}\left(\begin{array}{c}
-M, M+1+g^{2} \gamma_{0}, \frac{1}{2}+i u+\frac{i}{\sqrt{2}} g+\frac{1}{4} g^{2} \gamma_{0} \\
1+i \sqrt{2} g+\frac{1}{2} g^{2} \gamma_{0}, 1+\frac{1}{2} g^{2} \gamma_{0}
\end{array}\right)
$$

Expanding this in a Taylor series with respect to the 't Hooft coupling, we find for the two-loop correction itself

$$
Q_{1}(u)=b_{1} Q_{0}(u)+\left.\frac{1}{4}\left(2 \gamma_{0} \partial_{\delta_{1}}-\partial_{\delta_{2}}^{2}-\partial_{\delta_{3}}^{2}\right){ }_{3} F_{2}\left(\begin{array}{c}
-M, M+1+2 \delta_{1}, \frac{1}{2}+i u+\delta_{2} \\
1+\delta_{1}+\delta_{2}+\delta_{3}, 1+\delta_{2}-\delta_{3}
\end{array} \mid 1\right)\right|_{\delta_{i}=0}
$$

where we chose a spin-dependent form of the perturbative expansion of the normalization constant $N(g) \simeq 1+g^{2} b_{1}(M)$ with

$$
b_{1}(M)=4 S_{1}^{2}+S_{2}-2 S_{1} \widetilde{S}_{1}
$$

in order to reduce the degree of $Q_{1}$ in accordance with the definition (2.3) such that $\operatorname{deg} Q_{1}=\left(\operatorname{deg} Q_{0}-2\right)$. Here and below the nested harmonic sums (A.4) appear as functions of two arguments

$$
S_{a_{1}, a_{2}, \ldots} \equiv S_{a_{1}, a_{2}, \ldots}(M), \quad \widetilde{S}_{a_{1}, a_{2}, \ldots} \equiv S_{a_{1}, a_{2}, \ldots}(2 M) .
$$

\footnotetext{
${ }^{3}$ We summarize their basic properties in Appendix B.
} 
The Baxter function for twist-two operators in the basis of continuous Hahn polynomials has been obtained analytically to three-loop order in [23. However, as can be seen from Eq. (3.4) the number of deformation terms arising is quite substantial. This calls for a quest to find a more concise representation. To this end one notices that the Baxter function for the ground state is symmetric under the reflection $u \rightarrow-u$, which is however not transparent in the representation in terms of Hahn polynomials (3.1) but becomes explicit in the basis of the Wilson polynomials 4

$$
Q_{0}(u)={ }_{4} F_{3}\left(\begin{array}{c}
-\frac{M}{2}, \frac{M+1}{2}, \frac{1}{2}+i u, \frac{1}{2}-i u \mid \\
1,1, \frac{1}{2}
\end{array} \mid\right.
$$

Analogously to the previous consideration, matching the two-loop Baxter equation to the equation for Wilson polynomials (B.7), we find the two-loop solution

$$
Q(u)=N(g)_{4} F_{3}\left(\begin{array}{c}
-\frac{M}{2}, \frac{M+1}{2}+\frac{1}{2} g^{2} \gamma_{0}, \frac{1}{2}+i u, \frac{1}{2}-i u \\
1+\frac{i}{\sqrt{2}} g+\frac{1}{4} g^{2} \gamma_{0}, 1-\frac{i}{\sqrt{2}} g+\frac{1}{4} g^{2} \gamma_{0}, \frac{1}{2}
\end{array} \mid 1\right)
$$

up to an overall coupling-dependent normalization constant $N(g)$. Expanding this result to order $g^{2}$ yields the two-loop Baxter polynomial $Q_{1}(u)$ in Wilson basis

$$
Q_{1}(u)=a_{1}(M) Q_{0}(u)+\left.\frac{1}{4}\left(2 \gamma_{0} \partial_{\delta_{1}}-\partial_{\delta_{2}}^{2}\right){ }_{4} F_{3}\left(\begin{array}{c}
-\frac{M}{2}, \frac{M+1}{2}+\delta_{1}, \frac{1}{2}+i u, \frac{1}{2}-i u \\
1+\delta_{1}+\delta_{2}, 1-\delta_{2}, \frac{1}{2}
\end{array} \mid 1\right)\right|_{\delta_{1}, \delta_{2}=0},
$$

where the normalization constant is $N(g) \simeq 1+g^{2} a_{1}(M)$ with

$$
a_{1}(M)=3 S_{1}^{2}+S_{2}+S_{-2}-2 S_{1} \widetilde{S}_{1} .
$$

A few comments are in order concerning the relation of this representation to the one in terms of continuous Hahn polynomials. First of all, there is one deformation less. As a consequence, the number of polynomial contributions in higher loops will drastically decrease. Second, the deformed parts in the two representations are not identical and hence their degree reduction coefficients multiplying the leading order solution differ as well. However, it should be noted that these degree reduction coefficients can, in both cases, not contribute to the anomalous dimension by symmetry arguments. Although less obvious, the same is true for higher-loop contributions.

Let us now turn to higher loops contributions and use the finding of this section to devise an efficient formalism to determine perturbative solutions. We will present the results both in the basis of Wilson and continuous Hahn polynomials.

\section{Polynomial contributions}

To start with, as we observed in the previous section in order to tackle higher order corrections to the Baxter function it suffices to introduce a doubly-deformed function and

\footnotetext{
${ }^{4}$ We briefly review them in Appendix B,
} 
its derivatives with respect to the deformation parameters

$$
\left.T_{(p, q)} \equiv \partial_{\delta_{1}}^{p} \partial_{\delta_{2}}^{2 q}{ }_{4} F_{3}\left(\begin{array}{c}
-\frac{M}{2}, \frac{M+1}{2}+\delta_{1}, \frac{1}{2}+i u, \frac{1}{2}-i u \\
1+\delta_{1}+\delta_{2}, 1-\delta_{2}, \frac{1}{2}
\end{array} \mid 1\right)\right|_{\delta_{1}=\delta_{2}=0} .
$$

Then a straightforward scheme presents itself for the construction of the $\ell-$ th order function $Q_{\ell}(u)$. That is, $Q_{\ell}(u)$ is a linear superposition of the structures $T_{\left(\ell_{1}, \ell_{2}\right)}$ with $\ell_{1}+\ell_{2} \leq \ell$ accompanied by degree- $d$ transcendental numbers of $d=2 \ell-2 \ell_{2}-\ell_{1}$ and a $Q_{0}$-proportional term, such that the degree of $Q_{\ell}$ is reduced to $\operatorname{deg} Q_{\ell}=\left(\operatorname{deg} Q_{0}-2\right)$.

Analogously, in the basis of continuous Hahn polynomials we have a triple- deformed function

$$
T_{(p, q, r)}=\left.\partial_{\delta_{1}}^{p} \partial_{\delta_{2}}^{q} \partial_{\delta_{3} 3}^{r} F_{2}\left(\begin{array}{c}
-M, M+1+\delta_{1}, \frac{1}{2}+i u+\delta_{2} \\
1+\delta_{1}+\delta_{2}+\delta_{3}, 1+\delta_{2}-\delta_{3}
\end{array} \mid 1\right)\right|_{\delta_{1}=\delta_{2}=\delta_{3}=0} .
$$

In the basis of Hahn polynomials there are more possible deformations than in the Wilson basis. As a consequence the number of terms at each order of the perturbative series is also considerably increased. As was shown in Ref. [23] at three loops, a simple counting 5 gives a total number of eleven terms. Changing the representation from ${ }_{3} F_{2} \rightarrow{ }_{4} F_{3}$ reduces the number of contributions to the Baxter function by almost half. At four-loop order this effect will decrease the number of contributions from 36 to 15 terms, as demonstrated below.

In the following we will divide the contributions to the Baxter functions into polynomial and non-polynomial contributions

$$
Q_{\ell}(u)=Q_{\ell}^{(p)}(u)+Q_{\ell}^{(n p)}(u),
$$

respectively. The terminology used here needs clarification. Of course, at any given order of perturbation theory, the Baxter equation is polynomial. However, it consists of two types of terms, the first one explicitly polynomial in the spectral parameter $u$ and the other containing inverse powers of the spectral parameter $u^{ \pm}$accompanying Baxter polynomials and thus appearing superficially non-polynomial. Indeed the inverse powers of $u$ conspire to cancel in the sum of the latter such that the net result is polynomial as it should. However we choose to split the Baxter function according to this nomenclature inherited from their source in the equation.

\subsection{Wilson basis}

Following the strategy outlined above we find first the polynomial part of the perturbative Baxter function in the Wilson basis. First, the three-loop Baxter function $Q_{2}^{(p)}$ reads

$$
Q_{2}^{(p)}(u)=a_{2} Q_{0}(u)+\frac{1}{2}\left(\gamma_{1}+a_{1} \gamma_{0}\right) T_{(1,0)}-\frac{1}{8}\left(K_{2}+2 a_{1}\right) T_{(0,1)}
$$

\footnotetext{
${ }^{5}$ Note, that for this representation, there is a term $T_{(0,0,3)}$, which has a third- order derivative w.r.t. the deformation parameter.
} 


$$
+\frac{1}{8} \gamma_{0}^{2} T_{(2,0)}-\frac{1}{8} \gamma_{0} T_{(1,1)}+\frac{1}{96} T_{(0,2)}
$$

where the transcendental coefficient $K_{2}$ of degree two is a linear superposition of anomalous dimensions and inhomogeneities introduced in Appendix $\mathrm{A}$

$$
K_{2}=\alpha+\frac{3}{4} \gamma_{0}^{2}=\frac{1}{2} \gamma_{0}^{2}-S_{-2}
$$

and the normalization function $a_{2}=a_{2}(M)$ reducing the degree of the higher-loop polynomial depends on the non-polynomial contribution computed later in Section 5.

At four-loop order a further transcendental function arises from the Baxter equation, see Appendix A, from the expansion coefficients of the dressing factors (2.9). They appear in a certain combination with a degree of transcendentality four,

$$
K_{4}=-6 \alpha^{2}+24 \chi-\varepsilon \gamma_{0}+\delta \gamma_{0}^{2}+\frac{1}{4} \gamma_{0}^{4}+10 \gamma_{0} \gamma_{1}
$$

So that finally the polynomial part of the four-loop Baxter function is

$$
\begin{aligned}
& Q_{3}^{(p)}(u)=a_{3} Q_{0}(u)+\frac{1}{2}\left(\gamma_{2}+a_{1} \gamma_{1}+a_{2} \gamma_{0}\right) T_{(1,0)}-\frac{1}{48}\left(K_{4}+3 \zeta_{3} \gamma_{0}+6 a_{1} K_{2}+12 a_{2}\right) T_{(0,1)} \\
& \quad+\frac{1}{8}\left(2 \gamma_{1} \gamma_{0}+a_{1} \gamma_{0}^{2}\right) T_{(2,0)}-\frac{1}{8}\left(\gamma_{1}+a_{1} \gamma_{0}+\frac{1}{2} K_{2} \gamma_{0}\right) T_{(1,1)}+\frac{1}{192}\left(\gamma_{0}^{2}+2 K_{2}+2 a_{1}\right) T_{(0,2)} \\
& \quad+\frac{1}{48} \gamma_{0}^{3} T_{(3,0)}-\frac{1}{32} \gamma_{0}^{2} T_{(2,1)}+\frac{1}{192} \gamma_{0} T_{(1,2)}-\frac{1}{5760} T_{(0,3)},
\end{aligned}
$$

with the degree-reducing coefficient $a_{3}$. In the last equation, the term proportional to $\zeta_{3}$ stems from the dressing factor.

\subsection{Hahn basis}

Let us also include for completeness the three-loop Baxter function obtained in [23], converted to the notation of Eq. (4.2) and rescaled coupling,

$$
\begin{aligned}
Q_{2}^{(p)}(u)= & b_{2}(M) Q_{0}(u)+\frac{1}{2}\left(\gamma_{1}+b_{1} \gamma_{0}\right) T_{(1,0,0)}-\frac{1}{8}\left(K_{2}+2 b_{1}\right)\left(T_{(0,2,0)}+T_{(0,0,2)}\right) \\
& +\frac{1}{8} \gamma_{0}^{2} T_{(2,0,0)}-\frac{1}{8} \gamma_{0}\left(T_{(1,2,0)}+T_{(1,0,2)}+\frac{1}{3} T_{(0,0,3)}\right) \\
& +\frac{1}{96}\left(6 T_{(0,2,2)}+T_{(0,4,0)}+T_{(0,0,4)}\right) .
\end{aligned}
$$

The appearing functions are the two-loop normalization constant for the Hahn basis $b_{1}$ given in (3.5) and the transcendental function $K_{2}$ in (4.5). We omit the precise structure of the normalization constant $b_{2}(M)$, as it will be of no use for us.

In the basis of continuous Hahn polynomials the four-loop result is quite lengthy and reads in the conventions of Eq. (4.2),

$$
\begin{aligned}
Q_{3}^{(p)}= & b_{3} Q_{0}(u)+\frac{1}{2}\left(\gamma_{2}+b_{1} \gamma_{1}+b_{2} \gamma_{0}\right) T_{(1,0,0)} \\
& -\frac{1}{48}\left(K_{4}+3 \zeta_{3} \gamma_{0}+6 b_{1} K_{2}+12 b_{2}\right)\left(T_{(0,2,0)}+T_{(0,0,2)}\right) \\
& +\frac{1}{192} K_{2}\left(T_{(0,0,4)}+T_{(0,4,0)}+6 T_{(0,2,2)}\right) \\
& -\frac{1}{16} \gamma_{0}\left(K_{2}-2 \gamma_{1}+2 b_{1}\right)\left(T_{(1,2,0)}+\frac{1}{3} T_{(0,3,0)}+T_{(1,0,2)}\right)
\end{aligned}
$$




$$
\begin{aligned}
& +\frac{1}{8} \gamma_{0}\left(\gamma_{1}+b_{1} \gamma_{0}\right) T_{(2,0,0)}+\frac{1}{192}\left(2 b_{1}+K_{2}\right)\left(T_{(0,0,4)}+T_{(0,4,0)}+6 T_{(0,2,2)}\right) \\
& -\frac{1}{5760}\left(T_{(0,0,6)}+T_{(0,6,0)}+15 T_{(0,2,4)}+15 T_{(0,4,2)}\right) \\
& +\frac{1}{32} \gamma_{0}\left(T_{(1,2,2)}+\frac{1}{6} T_{(1,0,4)}+\frac{1}{3} T_{(0,3,2)}+\frac{1}{6} T_{(1,4,0)}+\frac{1}{15} T_{(0,5,0)}\right) \\
& -\frac{1}{32} \gamma_{0}^{2}\left(T_{(2,0,2)}+T_{(2,2,0)}+\frac{1}{6} T_{(0,4,0)}-\frac{1}{6} T_{(0,0,4)}+\frac{2}{3} T_{(1,3,0)}\right)+\frac{1}{48} \gamma_{0}^{3} T_{(3,0,0)} .
\end{aligned}
$$

Again, we omit the definition of the normalization $b_{3} . K_{4}$ is determined by (4.6). These expressions for the three- and four-loop Baxter functions (4.8) and (4.9) in the Hahn basis should be compared to their Wilson basis counterparts in Eqs. (4.4) and (4.7), respectively. It is apparent that the Wilson basis enormously simplifies higher- loop computations and should be the method of choice for subsequent loop-orders.

\section{Non-polynomial contributions}

In order to complete the solution to the Baxter equation we have to address the nonpolynomial contributions. According to the nomenclature of Section 4 , we collectively label all inhomogeneities of the form $\left(u^{ \pm}\right)^{-k} Q_{\ell}(u)$ with $k>1$ as non-polynomial. Recall however, that the transfer matrix (2.11) is chosen in such a way, that it compensates these non-polynomialities and in the sum of all these contributions at a given loop-order the polynomiality is restored.

Non-polynomial inhomogeneities in the Baxter equation of twist-two operators appear for the first time at three-loop order, while wrapping effects set in at four loops. According to our choice of splitting in (4.3) we will complete the three-loop Baxter function by obtaining the term $Q_{\ell=2}^{(n p)}$ in an novel form. The non-polynomial contributions at threeloop have already been found in [23]. However, the representation used there is given in terms of Stirling numbers, which complicates the computation of anomalous dimension. Therefore we will present a novel representation of these terms here, which is based solely on Mellin transform techniques of the $\left(u^{ \pm}\right)^{-k}$ with $k>1$. The procedure is different to the one presented in [23], as there the effective polynomial of all non-polynomial terms has been expressed in terms of Stirling numbers. Successively its contribution to the Baxter function has been obtained by Mellin transform 6 . What will be different in our novel representation is that we give the Mellin transform of all single non-polynomialities. This results in a toolbox, which allows to simply construct the solution from a general set of expressions. The advantage is, that the final representation does not depend on complicated coefficient functions involving Stirling numbers, rendering the computation of anomalous dimension more feasible.

Before we turn to the explanation of the method used in our calculation, let us summarize the results of our analysis in this section. The non-polynomial contributions to the three- and four-loop Baxter functions are given by the following expressions

$$
Q_{2}^{(n p)}(u)=\sum_{k=0}^{M} 2 \Re \mathrm{e}\left[P_{k}(u)\right] R_{k}(M) r_{2, k}^{(n p)}(M),
$$

\footnotetext{
${ }^{6}$ This procedure is given as Lemma 1 in 23.
} 


$$
Q_{3}^{(n p)}(u)=\sum_{k=0}^{M} 2 \Re \mathrm{e}\left[P_{k}(u)\right] R_{k}(M)\left(r_{3, k}^{(n p p)}(M)+r_{3, k}^{(p n p)}(M)\right)
$$

with expansion coefficients determined in Eqs. (5.13), and (5.39), (5.50), (5.51) respectively. These expressions complete the three- and four-loop Baxter function, which is given by the sum of (4.4) and (5.1), and (4.7) and (5.2), respectively. The functions used in the representation of the non-polynomial parts are given by

$$
R_{k}(M)=\frac{(-1)^{k}}{(k !)^{2}} \frac{\Gamma(M+1+k)}{\Gamma(M+1-k)}, \quad P_{k}(u)=\frac{\Gamma\left(k+\frac{1}{2}+i u\right)}{k ! \Gamma\left(\frac{1}{2}+i u\right)} .
$$

Details of the computation are presented in the following subsections, while the complete dissection of the Mellin techniques is deferred to Appendix D.

\subsection{Three loops}

At three loops order, the non-polynomial contribution $U_{2}(u)$ to the Baxter equation

$$
\mathfrak{B}\left[Q_{2}\right]=\cdots+U_{2}\left[Q_{0}\right]
$$

reads

$$
U_{2}\left[Q_{0}\right]=\frac{1}{16\left(u^{+}\right)^{2}}\left[Q_{0}(u+i)-Q_{0}(u)\right]+\frac{i \gamma_{0}^{+}}{4 u^{+}} Q_{0}(u+i)+\text { c.c. . }
$$

The procedure for finding a closed solution to this equation via the Mellin transform technique together with a complete set of building blocks required for generic higher order analysis of non-polynomial contributions is presented in Appendix D. We refer to it for a complete list of definitions of objects arising in this calculation. Below we merely assemble specific terms at three-loop order.

Extracting the first, constant term from $Q_{0}$ accompanying $\gamma_{0}$ in Eq. (5.5) and introducing a new function $\widetilde{Q}_{0}$ via Eq. (D.73), we can decompose $U_{2}$ into a sum of two terms with each of them being separately polynomial. Then, making use of Eqs. (D.90), (D.93) and (D.95) we can obtain solutions to Eq. (5.4) stemming separately from both combinations of non-polynomial contributions.

The first inhomogeneity in $U_{2}$ is of the form of (D.90) with $L=0$, i.e.,

$$
\frac{1}{u^{+}} \widetilde{Q}_{0}(u+i)-\frac{1}{u^{-}} \widetilde{Q}_{0}(u-i)=i \sum_{p=1}^{M} \frac{2 \Re \mathrm{e}\left[P_{p-1}(u)\right]}{p} R_{p}(M) .
$$

Then, matching the right-hand side of this equation to Eq. (D.18) provides via Eq. (D.25) the contribution of this inhomogeneous term to the Baxter function $Q_{2}(u)$, which reads

$$
i \sum_{k=0}^{M} 2 \Re \mathrm{e}\left[P_{k}(u)\right] R_{k}(M) S_{3}(k)
$$


up to an overall factor $\frac{i}{8} \gamma_{0}= \pm \frac{i}{4} \gamma_{0}^{ \pm}$accompanying (5.6) in Eq. (5.5) .

Now turning to the second inhomogeneity in $U_{2}$, one notices that it is given as a linear combination of Eqs. (D.90), (D.91) and (D.93) with $L=1$ and $\widetilde{Q}_{0}\left( \pm \frac{i}{2}\right)=0$

$$
\begin{aligned}
\frac{1}{\left(u^{+}\right)^{2}} \widetilde{Q}_{0}(u+i)+\frac{1}{\left(u^{-}\right)^{2}} \widetilde{Q}_{0}(u-i) & =-i Q_{0}^{\prime}\left(\frac{i}{2}\right) \sum_{p=M+1}^{\infty} \frac{2 \Re \mathrm{e}\left[P_{p-1}(u)\right]}{p} \\
& +\sum_{p=1}^{M} \frac{2 \Re \mathrm{e}\left[P_{p-1}(u)\right]}{p} \sum_{k=1}^{p} \frac{R_{k}(M)}{k}, \\
\frac{1}{\left(u^{+}\right)^{2}} \widetilde{Q}_{0}(u)+\frac{1}{\left(u^{-}\right)^{2}} \widetilde{Q}_{0}(u) & =-i Q_{0}^{\prime}\left(-\frac{i}{2}\right) \sum_{p=M+1}^{\infty} \frac{2 \Re \mathrm{e}\left[P_{p-1}(u)\right]}{p} \\
& -\sum_{p=1}^{M} \frac{2 \Re \mathrm{e}\left[P_{p-1}(u)\right]}{p} \sum_{k=1}^{p} R_{k}(M) Z_{1}(p, k),
\end{aligned}
$$

and (D.95) with $L=0$

$$
\frac{1}{u^{+}}-\frac{1}{u^{-}}=-i \sum_{p=1}^{\infty} \frac{2 \Re \mathrm{e}\left[P_{p-1}(u)\right]}{p}
$$

respectively. Then, the sought-after combination of terms is

$$
\begin{aligned}
\frac{1}{\left(u^{+}\right)^{2}}\left(\widetilde{Q}_{0}(u+i)\right. & \left.-\widetilde{Q}_{0}(u)\right)+\frac{1}{\left(u^{-}\right)^{2}}\left(\widetilde{Q}_{0}(u-i)-\widetilde{Q}_{0}(u)\right)+2 i \gamma_{0}\left(\frac{1}{u^{+}}-\frac{1}{u^{-}}\right) \\
& =\sum_{p=1}^{M} \frac{2 \Re \mathrm{e}\left[P_{p-1}(u)\right]}{p} \sum_{k=1}^{p} R_{k}(M) Z_{1}(p, k-1) \\
& -i\left(Q_{0}^{\prime}\left(\frac{i}{2}\right)-Q_{0}^{\prime}\left(-\frac{i}{2}\right)\right) \sum_{p=M+1}^{\infty} \frac{2 \Re \mathrm{e}\left[P_{p-1}(u)\right]}{p}+2 \gamma_{0} \sum_{p=1}^{\infty} \frac{2 \Re \mathrm{e}\left[P_{p-1}(u)\right]}{p}
\end{aligned}
$$

And since $i Q_{0}^{\prime}\left( \pm \frac{i}{2}\right)= \pm \gamma_{0}$, the contribution of the infinite series $\sim \sum_{p=M+1}^{\infty}$ cancels between the last two terms and the result takes a polynomial form

$$
\begin{aligned}
\frac{1}{\left(u^{+}\right)^{2}}\left(\widetilde{Q}_{0}(u+i)\right. & \left.-\widetilde{Q}_{0}(u)\right)+\frac{1}{\left(u^{-}\right)^{2}}\left(\widetilde{Q}_{0}(u-i)-\widetilde{Q}_{0}(u)\right)+2 i \gamma_{0}\left(\frac{1}{u^{+}}-\frac{1}{u^{-}}\right) \\
& =\sum_{p=1}^{M} \frac{2 \Re \mathrm{e}\left[P_{p-1}(u)\right]}{p}\left\{\sum_{k=1}^{p} R_{k}(M) Z_{1}(p, k-1)+2 \gamma_{0}\right\} .
\end{aligned}
$$

Combining this result with the one found earlier in Eq. (5.7) multiplied by its proper relative coefficient in Eq. (5.5) and identifying the expansion coefficients in the summand with $B_{k}$ 
of Eq. (D.24), we can immediately write down the contribution of both inhomogeneous terms to $Q_{2}$ as

$$
16 \sum_{k=0}^{M} 2 \Re \mathrm{e}\left[P_{k}(u)\right] R_{k}(M) r_{2, k}^{(n p)}(M)
$$

with $r_{2, k}^{(n p)}$, introduced in Eq. (D.25), taking the following explicit form

$$
\begin{aligned}
r_{2, k}^{(n p)}(M) & =\frac{1}{16}\left(\widehat{V}_{3,1}(k)+\widetilde{V}_{3,1}(k)+2 \gamma_{0}\left(W_{3}(k)-S_{3}(k)\right)\right) \\
& =\frac{1}{16}\left(V_{3,1}(k)+2 \gamma_{0}\left(W_{3}(k)-S_{3}(k)\right)\right)
\end{aligned}
$$

determined in terms of the following sums

$$
\begin{aligned}
& W_{a}(k)=\sum_{l=1}^{k} \frac{1}{l^{a} R_{l}(M)}, \\
& V_{a, b}(k)=\sum_{r=1}^{k} \frac{1}{r^{a} R_{r}(M)} \sum_{m=1}^{r} R_{m}(M) Z_{b}(r, m-1), \\
& \widehat{V}_{a, b}(k)=\sum_{r=1}^{k} \frac{1}{r^{a} R_{r}(M)} \sum_{m=1}^{r} \frac{R_{m}(M)}{m} Z_{b-1}(r, m-1), \\
& \widetilde{V}_{a, b}(k)=\sum_{r=1}^{k} \frac{1}{r^{a} R_{r}(M)} \sum_{m=1}^{r} R_{m}(M) Z_{b}(r, m),
\end{aligned}
$$

with $Z_{b}$ introduced in Eq. (D.87). Restoring the overall coefficient $\frac{1}{16}$ of (15.11) in Eq. (15.5), we recognize (5.12) as Eq. (5.1).

\subsection{Four loops}

At four loops the number of non-polynomial contributions is greater, however, due to the perturbative iteration, the contribution of inhomogeneities due to the two-loop Baxter function $Q_{1}$ has the same form as in Eq. (5.5), i.e., $U_{2}\left[Q_{1}\right]$. The complete set of nonpolynomial inhomogeneities then consists of three terms,

$$
\mathfrak{B}\left[Q_{3}\right]=\cdots+U_{2}\left[Q_{1}\right]+U_{3}\left[Q_{0}\right]+U_{1}\left[Q_{2}^{(n p)}\right]
$$

where $U_{1}\left[Q_{2}^{(n p)}\right]$ arises from non-polynomial contributions to the three- loop Baxter function $Q_{2}$ computed above and has the form

$$
\begin{aligned}
U_{1}\left[Q_{2}^{(n p)}\right]= & \left(\frac{1}{2}-i \gamma_{0}^{+} u^{+}\right) Q_{2}^{(n p)}(u+i)+\left(\frac{1}{2}-i \gamma_{0}^{-} u^{-}\right) Q_{2}^{(n p)}(u-i) \\
& -\left(1+(2 M+1) \gamma_{0}^{+}\right) Q_{2}^{(n p)}(u) .
\end{aligned}
$$


While $U_{3}$ is another novel non-polynomial function of the leading order $Q_{0}$,

$$
\begin{aligned}
U_{3}\left[Q_{0}\right] & =\left\{\frac{1}{32\left(u^{+}\right)^{4}}\left(Q_{0}(u+i)-Q_{0}(u)\right)+\frac{i \gamma_{0}^{+}}{16\left(u^{+}\right)^{3}}\left(Q_{0}(u+i)+Q_{0}(u)\right)\right. \\
& \left.+\frac{i B_{1}^{+}}{24 u^{+}} Q_{0}(u+i)-\frac{i}{4 u^{+}} \gamma_{0}^{+}\left(\alpha^{+}+\left(\gamma_{0}^{+}\right)^{2}\right) Q_{0}(u)\right\}+ \text { c.c. }
\end{aligned}
$$

where

$$
B_{1}^{ \pm}=10\left(\gamma_{0}^{ \pm}\right)^{3}+4 \gamma_{1}^{ \pm}+6 \gamma_{0}^{ \pm} \alpha^{ \pm}-2 \gamma_{0}^{ \pm} \delta^{ \pm}+\varepsilon^{ \pm} .
$$

Let us address all three contributions in turn, starting with the latter.

\subsubsection{Inhomogeneity $U_{3}$}

Following the methodology developed at three-loop order, we split the inhomogeneity $U_{3}$ into a sum of terms whose series representations can be matched into the generic types analyzed in Appendix D. According to results given there, the inhomogeneity in Eq. (5.20) is a linear combination of Eqs. (D.90), (D.93) with $L=0,2,3$ and $\widetilde{Q}_{0}\left( \pm \frac{i}{2}\right)=0$, namely,

$$
\begin{aligned}
\frac{1}{\left(u^{+}\right)^{4}}\left(\widetilde{Q}_{0}(u+i)-\widetilde{Q}_{0}(u)\right)+\frac{1}{\left(u^{-}\right)^{4}}\left(\widetilde{Q}_{0}(u-i)-\widetilde{Q}_{0}(u)\right) \\
=-\sum_{p=1}^{M} \frac{2 \Re \mathrm{e}\left[P_{p-1}(u)\right]}{p} \sum_{k=1}^{p} R_{k}(M)\left(\frac{1}{2 k} Z_{2}(p, k-1)+\frac{1}{6} Z_{3}(p, k)\right) \\
+\sum_{p=M+1}^{\infty} \frac{2 \Re \mathrm{e}\left[P_{p-1}(u)\right]}{p}\left\{\frac{i}{2}\left(Q_{0}^{\prime}\left(-\frac{i}{2}\right)-Q_{0}^{\prime}\left(\frac{i}{2}\right)\right) Z_{2}(p)\right. \\
\left.\quad+\frac{1}{2}\left(Q_{0}^{\prime \prime}\left(-\frac{i}{2}\right)-Q_{0}^{\prime \prime}\left(\frac{i}{2}\right)\right) Z_{1}(p)+\frac{i}{6}\left(Q_{0}^{\prime \prime \prime}\left(-\frac{i}{2}\right)-Q_{0}^{\prime \prime \prime}\left(\frac{i}{2}\right)\right)\right\}
\end{aligned}
$$

multiplied by the factor of $\frac{1}{32}$. The term

$$
\begin{gathered}
\frac{i}{\left(u^{+}\right)^{3}}\left(\widetilde{Q}_{0}(u+i)+\widetilde{Q}_{0}(u)\right)-\frac{i}{\left(u^{-}\right)^{3}}\left(\widetilde{Q}_{0}(u-i)+\widetilde{Q}_{0}(u)\right) \\
=\sum_{p=1}^{M} \frac{2 \Re \mathrm{e}\left[P_{p-1}(u)\right]}{p} \sum_{k=1}^{p} R_{k}(M)\left(\frac{1}{k} Z_{1}(p, k-1)-\frac{1}{2} Z_{2}(p, k)\right) \\
-\sum_{p=M+1}^{\infty} \frac{2 \Re \mathrm{e}\left[P_{p-1}(u)\right]}{p}\left\{i\left(Q_{0}^{\prime}\left(-\frac{i}{2}\right)+Q_{0}^{\prime}\left(\frac{i}{2}\right)\right) Z_{1}(p)-\frac{1}{2}\left(Q_{0}^{\prime \prime}\left(-\frac{i}{2}\right)+Q_{0}^{\prime \prime}\left(\frac{i}{2}\right)\right)\right\}
\end{gathered}
$$

accompanied by $\frac{1}{16} \gamma_{0}^{+}$. And the terms

$$
\frac{i}{u^{+}} \widetilde{Q}_{0}(u+i)-\frac{i}{u^{-}} \widetilde{Q}_{0}(u-i)=-\sum_{p=1}^{M} \frac{2 \Re \mathrm{e}\left[P_{p-1}(u)\right]}{p} R_{p}(M),
$$




$$
\frac{i}{u_{+}} \widetilde{Q}_{0}(u)-\frac{i}{u_{-}} \widetilde{Q}_{0}(u)=\sum_{p=1}^{M} \frac{2 \Re \mathrm{e}\left[P_{p-1}(u)\right]}{p} \sum_{k=1}^{p-1} R_{k}(M),
$$

with the factors of $\frac{1}{24} B_{1}^{+}$and $-\frac{1}{4} \gamma_{0}^{+}\left[\left(\gamma_{0}^{+}\right)^{2}+\alpha^{+}\right]$, respectively. Further, for the completion of the last three equations we need as well Eq. (D.95) for $L=2$ times $\frac{i}{8} \gamma_{0}^{+}$, and for $L=0$ entering with $\frac{i}{24} B_{1}^{+}-\frac{i}{4} \gamma_{0}^{+}\left[\left(\gamma_{0}^{+}\right)^{2}+\alpha^{+}\right]$.

Combining all these expressions and considering contributions proportional to $Z_{2}(p)$, we find that the infinite series present in separate terms cancel between the two equations (5.22) and Eq. (D.95) for $L=2$ and their net result is equal to

$$
-\frac{1}{16} \gamma_{0}^{+} \sum_{p=1}^{M} \frac{2 \Re \mathrm{e}\left[P_{p-1}(u)\right]}{p} Z_{2}(p) .
$$

At the same time, the non-polynomial coefficients accompanying $Z_{1}(p)$ vanish since the leading order Baxter polynomial is an even function of the spectral parameter. Turning to the remaining two infinite-series contributions we deduce that they can be resummed into a concise expression such that the total inhomogeneity $U_{3}$ admits the following form with clearly separated polynomial terms

$$
\begin{aligned}
U_{3}\left[Q_{0}\right]= & -\frac{1}{32} \sum_{p=1}^{M} \frac{2 \Re \mathrm{e}\left[P_{p-1}(u)\right]}{p}\left\{\left[\frac{4}{3} B_{1}^{+}-8 \gamma_{0}^{+}\left(\left(\gamma_{0}^{+}\right)^{2}+\alpha^{+}\right)\right] R_{p}(M)\right. \\
+ & \sum_{k=1}^{p} R_{k}(M)\left[\frac{1}{6} Z_{3}(p, k-1)-2 \gamma_{0}^{+}\left(\frac{1}{k} Z_{1}(p, k-1)-\frac{1}{2} Z_{2}(p, k)\right)\right. \\
& \left.\left.\quad+8 \gamma_{0}^{+}\left(\left(\gamma_{0}^{+}\right)^{2}+\alpha^{+}\right)\right]+8\left(\gamma_{0}^{+} \alpha^{+}-8 \beta^{+}\right)+2 \gamma_{0}^{+} Z_{2}(p)\right\} \\
+ & \frac{i}{8}\left(\frac{1}{u^{+}}-\frac{1}{u^{-}}\right)\left(\varepsilon^{+}-2 \gamma_{0}^{+} \delta^{+}\right) .
\end{aligned}
$$

Here we used the identity

$$
\frac{1}{L !} Z_{L}(p, k-1)=\frac{1}{L !} Z_{L}(p, k)+\frac{1}{(L-1) !} \frac{Z_{L-1}(p, k-1)}{k}
$$

to simplify intermediate results. Even though there are remaining non-polynomial contributions, (the last line in Eq. (5.27)) that do not cancel on their own, they will after we add terms stemming from $U_{2}\left[Q_{1}\right]$ as will be demonstrated in the next section following the same lines of reasoning as in Section 5.1 upon the replacement $Q_{0}(u) \rightarrow Q_{1}(u)$.

\subsubsection{Inhomogeneity $U_{2}$}

To start with, we write the two-loop Baxter polynomial as

$$
Q_{1}(u)=\sum_{p=0}^{M} \Re \mathrm{e}\left[P_{p}(u)\right] R_{p}(M) \widetilde{r}_{1, p}(M),
$$


with (see Ref. [23])

$$
\widetilde{r}_{1, p}(M)=\widetilde{b}(M)+\gamma_{0}(M)\left(S_{1}(p+M)-S_{1}(M)-\frac{1}{2} S_{1}(p)\right)-S_{2}(p),
$$

and

$$
\widetilde{b}(M)=b_{1}(M)-S_{1}^{2}(M),
$$

where $b_{1}$ was introduced in Eq. (3.5), such that $\widetilde{r}_{1,0}(M)=Q_{1}\left(\frac{i}{2}\right)$. Then we split $Q_{1}$ into a constant piece and the rest $\widetilde{Q}_{1}(u)$ starting from $P_{p>0}(u)$

$$
Q_{1}(u)=\widetilde{r}_{1,0}(M)+\widetilde{Q}_{1}(u) .
$$

Then as in Section [5.1, the inhomogeneity $U_{2}\left[Q_{1}\right]$ is written as a sum of three terms: first, Eq. (5.6) multiplied by $\frac{i}{4} \gamma_{0}^{+}$, where we merely replace $Q_{0}$ by $Q_{1}$ and $R_{k}$ by $R_{1, k}=R_{k} \widetilde{r}_{1, k}$; the difference of Eqs. (5.8) and (5.9), both multiplied by $\frac{1}{16}$, with the same substitutions; and Eq. (5.10) accompanied by $\frac{i}{4} \gamma_{0}^{+} \widetilde{r}_{1,0}$.

In order to perform the reduction of the inhomogeneity $U_{2}$ to a polynomial form, it is sufficient to transform the summand of Eq. (5.29) to a form involving just the polynomial $P_{p}(u)$ itself rather than its real part, i.e., half the sum of $P_{p}(u)$ and $P_{p}(-u)$. In Mellin space $P_{p}(u)$ corresponds to a polynomial in $z^{n}$ alone, but not $(1-z)^{n}$. As can be observed easily, the terms proportional to $\widetilde{b}(M)$ and $\gamma_{0}(M)$ are even functions of the spectral parameter and, as a consequence, do not change after the substitution $u \rightarrow-u$. On the other hand, the term $\sim S_{2}(p)$ in the right-hand side of (5.30) does not have this property. Indeed, from Appendix D we have

$$
\sum_{p=0}^{M} P_{p}(-u) R_{p}(M) S_{2}(p)=\sum_{p=0}^{M} P_{p}(u) R_{p}(M)\left[V_{2,0}(p)-S_{2}(p)-2 S_{-2}(M)\right],
$$

and deduce a complimentary representation of the two-loop Baxter polynomial

$$
Q_{1}(u)=\sum_{p=0}^{M} P_{p}(u) R_{p}(M) r_{1, p}(M)
$$

with

$$
r_{1, p}(M)=\widetilde{b}(M)+\gamma_{0}(M)\left(S_{1}(p+M)-S_{1}(M)-\frac{1}{2} S_{1}(p)\right)-\frac{1}{2} V_{2,0}(p)+S_{-2}(M) .
$$

Separating the infinite-series contributions from $U_{2}$ of the form as in Eq. (5.27), we find that their coefficient conspire to give the same overall coefficient but with the opposite sign, such that $U_{2}\left[Q_{1}\right]$ can be cast in the form

$$
U_{2}\left[Q_{1}\right]=\frac{1}{16} \sum_{p=1}^{M} \frac{2 \Re \mathrm{e}\left[P_{p-1}(u)\right]}{p}\left\{\sum_{k=1}^{p} R_{k}(M) r_{1, k}(M) Z_{1}(p, k-1)\right.
$$




$$
\left.-4 \gamma_{0}^{+} R_{p}(M) r_{1, p}(M)+2 \varepsilon^{+}\right\}-\frac{i}{8}\left(\frac{1}{u^{+}}-\frac{1}{u^{-}}\right)\left(\varepsilon^{+}-2 \gamma_{0}^{+} \delta^{+}\right)
$$

Therefore, the result for the sum of the two inhomogeneities $U_{3}\left[Q_{0}\right]+U_{2}\left[Q_{1}\right]$ is free from non-polynomialities and reads

$$
\begin{gathered}
-\frac{1}{16} \sum_{p=1}^{M} \frac{\Re \mathrm{e}\left[P_{p-1}(u)\right]}{p}\left\{\left[\frac{4}{3} B_{1}^{+}-8 \gamma_{0}^{+}\left(\left(\gamma_{0}^{+}\right)^{2}+\alpha^{+}\right)+8 \gamma_{0}^{+} r_{1, p}(M)\right] R_{p}(M)\right. \\
+\sum_{k=1}^{p} R_{k}(M)\left[\frac{1}{6} Z_{3}(p, k-1)-2\left(\frac{1}{k} \gamma_{0}^{+}+r_{1, k}(M)\right) Z_{1}(p, k-1)\right. \\
\left.\left.+\gamma_{0}^{+} Z_{2}(p, k)+8 \gamma_{0}^{+}\left(\left(\gamma_{0}^{+}\right)^{2}+\alpha^{+}\right)\right]-16 C_{3,0}+2 \gamma_{0}^{+} Z_{2}(p)\right\},
\end{gathered}
$$

with $C_{3,0}$ combining the functions $C_{3,0}=4 \beta^{+}+\frac{1}{4} \varepsilon^{+}-\frac{1}{2} \gamma_{0}^{+} \alpha^{+}$. The corresponding contribution to $Q_{3}$ is then given by

$$
Q_{3}^{(n p p)}(u)=\sum_{k=0}^{M} 2 \Re \mathrm{e}\left[P_{k}(u)\right] R_{k}(M) r_{3, k}^{(n p p)}(M)
$$

where we give $r_{3, k}^{(n p p)}$ in the same order as the corresponding inhomogeneities appear in Eq. (5.37)

$$
\begin{aligned}
r_{3, k}^{(n p p)}(M)= & -\left[\frac{1}{24} B_{1}^{+}-\frac{1}{4} \gamma_{0}^{+}\left(\left(\gamma_{0}^{+}\right)^{2}+\alpha^{+}\right)\right] S_{3}(k)-\frac{1}{4} \gamma_{0}^{+} \widehat{S}_{3,1}(k)-\frac{1}{192} V_{3,3}(k) \\
& +\frac{1}{16} \gamma_{0}^{+} \widehat{V}_{3,2}(k)+\frac{1}{16} V_{3,1,1}(k)-\frac{1}{32} \gamma_{0}^{+} \widetilde{V}_{3,2}(k) \\
& -\frac{1}{4} \gamma_{0}^{+}\left(\left(\gamma_{0}^{+}\right)^{2}+\alpha^{+}\right) \widetilde{V}_{3,0}(k)+\frac{1}{2} C_{3,0} W_{3}(k)-\frac{1}{16} \gamma_{0}^{+} W_{3,2}(k) .
\end{aligned}
$$

It is expressed via the following nested sums

$$
\begin{aligned}
\widehat{S}_{a, b}(k) & =\sum_{l=1}^{k} \frac{1}{l^{a}} r_{b, l}(M), \\
W_{a, b}(k) & =\sum_{l=1}^{k} \frac{1}{l^{a} R_{l}(M)} Z_{b}(l), \\
\widehat{V}_{a, b, c}(k) & =\sum_{r=1}^{k} \frac{1}{r^{a} R_{r}(M)} \sum_{m=1}^{r} \frac{R_{c, m}(M)}{m} Z_{b-1}(r, m-1), \\
\widetilde{V}_{a, b, c}(k) & =\sum_{r=1}^{k} \frac{1}{r^{a} R_{r}(M)} \sum_{m=1}^{r} R_{c, m}(M) Z_{b}(r, m), \\
V_{a, b, c}(k) & =\sum_{r=1}^{k} \frac{1}{r^{a} R_{r}(M)} \sum_{m=1}^{r} R_{c, m}(M) Z_{b}(r, m-1) .
\end{aligned}
$$


These are related to the previously introduced sums (5.14) - (5.17) via

$$
W_{a}(k)=W_{a, 0}(k), \quad \widehat{V}_{a, b}(k)=\widehat{V}_{a, b, 0}(k), \quad \widetilde{V}_{a, b}(k)=\widetilde{V}_{a, b, 0}(k), \quad V_{a, b}(k)=V_{a, b, 0}(k) .
$$

\subsubsection{Inhomogeneity $U_{1}$}

Finally, we turn to the polynomial inhomogeneity (5.19) of the non-polynomial part of the three-loop Baxter function $Q_{2}^{(n p)}$. It can be represented as a sum

$$
U_{1}\left[Q_{2}^{(n p)}\right]=U_{1}^{[0]}\left[Q_{2}^{(n p)}\right]+U_{1}^{[1]}\left[Q_{2}^{(n p)}\right]
$$

with

$$
\begin{aligned}
& U_{1}^{[0]}\left[Q_{2}^{(n p)}\right]=\frac{1}{2}\left(Q_{2}^{(n p)}(u+i)+Q_{2}^{(n p)}(u-i)-2 Q_{2}^{(n p)}(u)\right) \\
& U_{1}^{[1]}\left[Q_{2}^{(n p)}\right]=-\gamma_{0}^{+}\left(i u^{+} Q_{2}^{(n p)}(u+i)-i u^{-} Q_{2}^{(n p)}(u-i)+(2 M+1) Q_{2}^{(n p)}(u)\right) .
\end{aligned}
$$

From this, it is immediate to find the corresponding contribution to $Q_{3}$ to be

$$
Q_{3}^{(p n p),[i]}(u)=\sum_{k=0}^{M} \Re \mathrm{e}\left[P_{k}(u)\right] R_{k}(M) r_{3, k}^{(p n p),[i]}(M), \quad(i=0,1)
$$

where

$$
\begin{aligned}
& r_{3, k}^{(p n p),[0]}(M)=\widehat{R}_{2, M}^{(n p)}(M)\left(\widetilde{b}(M)+W_{2}(k)\right)-\sum_{m=1}^{k} \frac{\widehat{R}_{2, m}^{(n p)}(M)}{m^{2} R_{m}(M)}, \\
& r_{3, k}^{(p n p),[1]}(M)=2 \gamma_{0}^{+} \sum_{p=1}^{k}\left\{\frac{2}{p+M} r_{2, p-1}^{(n p)}(M)-\frac{1}{p} r_{2, p}^{(n p)}(M)\right\},
\end{aligned}
$$

and

$$
\widehat{R}_{2, m}^{(n p)}(M)=\sum_{p=1}^{m} R_{2, p}^{(n p)}(M), \quad R_{2, p}^{(n p)}(M)=R_{p}(M) r_{2, p}^{(n p)}(M), \quad \widehat{R}_{2, M}^{(n p)}(M)=Q_{2}^{(n p)}\left(-\frac{i}{2}\right) .
$$

\section{Anomalous dimensions}

Making use of the explicit solution to the Baxter equation to four loops, we can immediately calculate its derivatives at the argument $u= \pm \frac{i}{2}$ (see, e.g., Appendix C) and find the corresponding anomalous dimensions by means of Eq. (2.2) expanded to the required order of perturbation theory. The results are 7

$$
\gamma_{0}=2 S_{1}
$$

\footnotetext{
${ }^{7}$ All results are given for even values of $M$. An analytical continuation to complex values can be found in 27.
} 


$$
\begin{aligned}
& \gamma_{1}=-S_{3}-S_{-3}+2 S_{-2,1}-2 S_{1}\left(S_{2}+S_{-2}\right), \\
& \gamma_{2}=S_{5}+3 S_{-5}-2 S_{-3} S_{2}+2 S_{-2} S_{3}-24 S_{-2,1,1,1}-6 S_{-4,1}-6 S_{-3,2}-6 S_{-2,3} \\
& +12 S_{-3,1,1}+12 S_{-2,1,2}+12 S_{-2,2,1}+\left(S_{2}+2 S_{1}^{2}\right)\left(3 S_{-3}+S_{3}-2 S_{-2,1}\right) \\
& +S_{1}\left(8 S_{-4}+S_{-2}^{2}+4 S_{2} S_{-2}+2 S_{2}^{2}+3 S_{4}-12 S_{-3,1}-10 S_{-2,2}+16 S_{-2,1,1}\right) \text {, } \\
& \gamma_{3}^{\text {(asy) }}=4 S_{-7}+6 S_{7}+2\left(S_{-3,1,3}+S_{-3,2,2}+S_{-3,3,1}+S_{-2,4,1}\right)+3\left(-S_{-2,5}\right. \\
& \left.+S_{-2,3,-2}\right)+4\left(S_{-2,1,4}-S_{-2,-2,-2,1}-S_{-2,1,2,-2}-S_{-2,2,1,-2}-S_{1,-2,1,3}\right. \\
& \left.-S_{1,-2,2,2}-S_{1,-2,3,1}\right)+5\left(-S_{-3,4}+S_{-2,-2,-3}\right)+6\left(-S_{5,-2}\right. \\
& \left.+S_{1,-2,4}-S_{-2,-2,1,-2}-S_{1,-2,-2,-2}\right)+7\left(-S_{-2,-5}+S_{-3,-2,-2}\right. \\
& \left.+S_{-2,-3,-2}+S_{-2,-2,3}\right)+8\left(S_{-4,1,2}+S_{-4,2,1}-S_{-5,-2}-S_{-4,3}\right. \\
& \left.-S_{-2,1,-2,-2}+S_{1,-2,1,1,-2}\right)+9 S_{3,-2,-2}-10 S_{1,-2,2,-2}+11 S_{-3,2,-2} \\
& +12\left(-S_{-6,1}+S_{-2,2,-3}+S_{1,4,-2}+S_{4,-2,1}+S_{4,1,-2}-S_{-3,1,1,-2}-S_{-2,2,-2,1}\right. \\
& -S_{1,1,2,3}-S_{1,1,3,-2}-S_{1,1,3,2}-S_{1,2,1,3}-S_{1,2,2,-2}-S_{1,2,2,2}-S_{1,2,3,1}-S_{1,3,1,-2} \\
& -S_{1,3,1,2}-S_{1,3,2,1}-S_{2,-2,1,2}-S_{2,-2,2,1}-S_{2,1,1,3}-S_{2,1,2,-2}-S_{2,1,2,2} \\
& -S_{2,1,3,1}-S_{2,2,1,-2}-S_{2,2,1,2}-S_{2,2,2,1}-S_{2,3,1,1}-S_{3,1,1,-2}-S_{3,1,1,2}-S_{3,1,2,1} \\
& \left.-S_{3,2,1,1}\right)+13 S_{2,-2,3}-14 S_{2,-2,1,-2}+15\left(S_{2,3,-2}+S_{3,2,-2}\right) \\
& +16\left(S_{-4,1,-2}+S_{-2,1,-4}-S_{-2,-2,1,2}-S_{-2,-2,2,1}-S_{-2,1,-2,2}-S_{-2,1,1,-3}\right. \\
& -S_{1,-3,1,2}-S_{1,-3,2,1}-S_{1,-2,-2,2}-S_{2,-2,-2,1}+S_{-2,1,1,-2,1}+S_{1,1,-2,1,-2} \\
& \left.+S_{1,1,-2,1,2}+S_{1,1,-2,2,1}\right)-17 S_{-5,2}+18\left(-S_{4,-3}-S_{6,1}+S_{1,-3,3}\right) \\
& +20\left(-S_{1,-6}-S_{1,6}-S_{4,3}+S_{-5,1,1}+S_{-4,-2,1}+S_{-3,-2,2}+S_{-2,-4,1}\right. \\
& \left.+S_{-2,-3,2}+S_{1,3,3}+S_{3,1,3}+S_{3,3,1}-S_{1,1,-2,3}-S_{1,2,-2,-2}-S_{2,1,-2,-2}\right) \\
& -21 S_{3,4}+22\left(S_{1,-2,-4}+S_{2,2,3}+S_{2,3,2}+S_{3,-2,2}+S_{3,2,2}\right)+23\left(-S_{-3,-4}\right. \\
& \left.-S_{5,2}+S_{2,-2,-3}\right)+24\left(-S_{-4,-3}+S_{1,-4,-2}-S_{1,-3,1,-2}-S_{1,1,1,4}-S_{1,1,4,1}\right. \\
& -S_{1,3,-2,1}-S_{1,4,1,1}-S_{3,-2,1,1}-S_{3,1,-2,1}-S_{4,1,1,1}+S_{-2,-2,1,1,1}+S_{-2,1,-2,1,1} \\
& +S_{1,-2,-2,1,1}+S_{1,-2,1,-2,1}+S_{1,1,-2,-2,1}+S_{1,1,1,-2,-2}+S_{1,1,2,-2,1}+S_{1,2,1,-2,1} \\
& \left.+S_{2,1,1,-2,1}\right)+25 S_{2,-3,-2}+26\left(-S_{2,5}+S_{1,4,2}+S_{2,4,1}+S_{4,1,2}+S_{4,2,1}\right) \\
& +28\left(S_{1,2,4}+S_{2,1,4}-S_{-3,1,-2,1}-S_{-2,1,-3,1}-S_{1,-2,1,-3}\right)+30 S_{-3,1,-3} \\
& +32\left(S_{1,5,1}+S_{5,1,1}-S_{-3,-2,1,1}-S_{-2,-3,1,1}-S_{1,-3,-2,1}-S_{1,-2,-3,1}\right. \\
& \left.-S_{2,2,-2,1}+S_{1,2,-2,1,1}+S_{2,1,-2,1,1}-S_{1,1,1,-2,1,1}\right)+36\left(S_{1,1,5}+S_{1,3,-3}\right. \\
& +S_{3,1,-3}-S_{1,1,-3,-2}-S_{1,1,-2,-3}-S_{1,1,2,-3}-S_{1,2,-2,2}-S_{1,2,1,-3}-S_{2,1,-2,2} \\
& \left.-S_{2,1,1,-3}\right)+38 S_{-3,-3,1}+40\left(-S_{1,-4,1,1}-S_{2,-3,1,1}+S_{1,1,1,-2,2}\right) \\
& -41 S_{3,-4}+42\left(-S_{2,-5}+S_{1,-4,2}+S_{1,-3,-3}\right)+44\left(S_{1,-5,1}+S_{2,-3,2}+S_{3,-3,1}\right) \\
& +46 S_{2,2,-3}+48 S_{1,1,-3,1,1}+60\left(S_{1,1,-5}-S_{1,1,-3,2}\right)+62 S_{2,-4,1}+64 S_{1,1,1,-3,1} \\
& +68\left(S_{1,2,-4}+S_{2,1,-4}-S_{1,2,-3,1}-S_{2,1,-3,1}\right)-72 S_{1,1,1,-4}-80 S_{1,1,-4,1} \\
& -\zeta_{3} S_{1}\left(S_{3}-S_{-3}+2 S_{-2,1}\right) \text {. }
\end{aligned}
$$


These agree with expressions found from explicit calculations of Feynman diagrams at one [4] and two loops [15], and three [17] and four-loop [25] results obtained with the use of numerical solution of Bethe equations and the principle of maximal transcendentality.

\section{Five-loop dressing and reciprocity}

Finally, let us partially address the five loop order, namely, the one stemming from the dressing phase $\Theta(u)$. The dressing part of the five-loop Baxter polynomial can be written in terms of contributions with decreasing transcendentality as

$$
Q_{4}^{(d)}(u)=\zeta_{3}\left(Q_{\zeta_{3}}^{(p)}(u)+Q_{\zeta_{3}}^{(n p)}\right)+\zeta_{5} Q_{\zeta_{5}}^{(p)}(u)
$$

where we decomposed the term accompanying $\zeta_{3}$ according to the nomenclature of polynomial and non-polynomial inhomogeneities. Their calculation in the Wilson basis echoes the one performed in the previous section and yields for polynomial contributions,

$$
\begin{aligned}
& Q_{\zeta_{5}}^{(p)}(u)=a_{4, \zeta_{5}} Q_{0}+\frac{5}{16} S_{1} T_{(0,1)}, \\
& Q_{\zeta_{3}}^{(p)}(u)=a_{4, \zeta_{3}}^{(p)} Q_{0}+c_{(1,0)} T_{(1,0)}+c_{(2,0)} T_{(2,0)}+c_{(0,1)} T_{(0,1)}+c_{(1,1)} T_{(1,1)}+c_{(0,2)} T_{(0,2)} .
\end{aligned}
$$

Here

$$
\begin{aligned}
& c_{(1,0)}=-\frac{1}{2} S_{1}^{4}-\frac{1}{2} S_{2} S_{1}^{2}+S_{1,1} S_{1}^{2}, \\
& c_{(2,0)}=\frac{1}{4} S_{1}^{3}-\frac{1}{4} S_{-2} S_{1}, \\
& c_{(0,1)}=-\frac{5}{8} S_{1}^{3}+\frac{1}{4} \widetilde{S}_{1} S_{1}^{2}-\frac{1}{4} S_{2} S_{1}-\frac{1}{16} S_{-3}-\frac{1}{16} S_{3}+\frac{1}{8} S_{1,-2}+\frac{1}{8} S_{1,2}+\frac{1}{8} S_{2,1}, \\
& c_{(1,1)}=-\frac{1}{8} S_{1}^{2}, \\
& c_{(0,2)}=\frac{1}{96} S_{1},
\end{aligned}
$$

and the degree-reducing constants are

$$
\begin{aligned}
a_{4, \zeta_{5}}= & -\frac{5}{4} S_{1}\left(S_{-2}+S_{2}\right) \\
a_{4, \zeta_{3}}= & -\frac{15}{4} S_{1}^{5}+4 \widetilde{S}_{1} S_{1}^{4}-\widetilde{S}_{1}^{2} S_{1}^{3}+\frac{13}{4} S_{-2} S_{1}^{3}-\frac{7}{4} S_{2} S_{1}^{3}+\widetilde{S}_{2} S_{1}^{3}+3 S_{1,1} S_{1}^{3}-S_{-3} S_{1}^{2}+ \\
& -S_{3} S_{1}^{2}-3 S_{-2} \widetilde{S}_{1} S_{1}^{2}+S_{2} \widetilde{S}_{1} S_{1}^{2}-2 \widetilde{S}_{1} S_{1,1} S_{1}^{2}-\frac{1}{2} S_{-2}^{2} S_{1}+\frac{1}{2} S_{2}^{2} S_{1}+S_{-2} \widetilde{S}_{1}^{2} S_{1}+ \\
& -S_{-4} S_{1}+\frac{5}{4} S_{-2} S_{2} S_{1}-S_{4} S_{1}-S_{-2} \widetilde{S}_{2} S_{1}+\frac{1}{4} S_{-3} S_{-2}+\frac{1}{4} S_{-3} S_{2}+\frac{1}{4} S_{-2} S_{3}+ \\
& +\frac{1}{4} S_{2} S_{3}-\frac{1}{2} S_{-2} S_{1,-2}-\frac{1}{2} S_{2} S_{1,-2}-\frac{1}{2} S_{-2} S_{1,2}-\frac{1}{2} S_{2} S_{1,2}-\frac{1}{2} S_{-2} S_{2,1}-\frac{1}{2} S_{2} S_{2,1} .
\end{aligned}
$$

Finally, the non-polynomial term, obeys the equation

$$
\mathfrak{B}\left[Q_{\zeta_{3}}^{(n p)}\right]=-i S_{1}^{2} U_{\zeta_{3}}\left[Q_{0}\right]
$$

with

$$
U_{\zeta_{3}}\left[Q_{0}\right]=\frac{Q_{0}(u+i)-Q_{0}(u)}{u^{+}}-\frac{Q_{0}(u-i)-Q_{0}(u)}{u^{-}}
$$




$$
=2 i \sum_{p=1}^{M} \frac{\Re \mathrm{e}\left[P_{p-1}\right](u)}{p} \sum_{k=1}^{p} R_{k}(M)
$$

The polynomial $Q_{\zeta_{3}}^{(n p)}$ can be computed according to the method spelled out above. It reads

$$
Q_{\zeta_{3}}^{(n p)}(u)=2 S_{1}^{2} \sum_{p=0}^{M} \Re \mathrm{e}\left[P_{p}(u)\right] \sum_{l=1}^{p} \frac{1}{l^{3}} \frac{R_{p}(M)}{R_{l}(M)} \sum_{k=1}^{l} R_{k}(M) .
$$

Substituting these findings in Eq. (2.2) expanded to fifth order in the 't Hooft coupling, we find the dressing part of the five-loop anomalous dimensions of twist-two operators,

$$
\gamma_{5}^{(\text {asy })}=\cdots+\zeta_{3} \gamma_{5}^{\zeta_{3}}+\zeta_{5} \gamma_{5}^{\zeta_{5}}
$$

with spin-dependent functions $\gamma_{5}^{\zeta_{3}}$ and $\gamma_{5}^{\zeta_{5}}$ obeying the principle of maximal transcendentality (as well as the absence of -1 indices in nested harmonic sums)

$$
\begin{aligned}
\gamma_{5}^{\zeta_{5}}= & \frac{5}{2} S_{-4}-\frac{5}{2} S_{4}-\frac{15}{2} S_{-3,1}-5 S_{-2,2}-\frac{5}{2} S_{1,-3}+\frac{5}{2} S_{1,3}+\frac{5}{2} S_{3,1}+ \\
& +10 S_{-2,1,1}+5 S_{1,-2,1} \\
\gamma_{5}^{\zeta_{3}}= & -S_{-6}+S_{6}+11 S_{-5,1}+4 S_{-4,-2}+20 S_{-4,2}+9 S_{-3,-3}+14 S_{-3,3}+ \\
& +4 S_{-2,-4}+4 S_{-2,4}+3 S_{1,-5}-3 S_{1,5}+4 S_{2,-4}-4 S_{2,4}-3 S_{3,3}-2 S_{4,-2}+ \\
& -4 S_{4,2}-3 S_{5,1}-32 S_{-4,1,1}-8 S_{-3,-2,1}-10 S_{-3,1,-2}-28 S_{-3,1,2}-28 S_{-3,2,1}+ \\
& -6 S_{-2,-3,1}-2 S_{-2,-2,2}-10 S_{-2,1,-3}-10 S_{-2,1,3}-6 S_{-2,2,-2}-12 S_{-2,2,2}+ \\
& -10 S_{-2,3,1}-20 S_{1,-4,1}-6 S_{1,-3,-2}-24 S_{1,-3,2}-8 S_{1,-2,-3}-10 S_{1,-2,3}-4 S_{1,1,-4}+ \\
& +4 S_{1,1,4}-2 S_{1,2,-3}+2 S_{1,2,3}+2 S_{1,3,-2}+2 S_{1,3,2}+2 S_{1,4,1}-14 S_{2,-3,1}-2 S_{2,-2,-2}+ \\
& -10 S_{2,-2,2}-2 S_{2,1,-3}+2 S_{2,1,3}+2 S_{2,3,1}-2 S_{3,-2,1}+2 S_{3,1,-2}+2 S_{3,1,2}+2 S_{3,2,1}+ \\
& +4 S_{4,1,1}+36 S_{-3,1,1,1}+4 S_{-2,-2,1,1}+8 S_{-2,1,-2,1}++12 S_{-2,1,1,-2}+12 S_{-2,1,1,2}+ \\
& +12 S_{-2,1,2,1}+12 S_{-2,2,1,1}+36 S_{1,-3,1,1}+8 S_{1,-2,-2,1}+8 S_{1,-2,1,-2}+20 S_{1,-2,1,2}+ \\
& +20 S_{1,-2,2,1}+16 S_{1,1,-3,1}+4 S_{1,1,-2,-2}+12 S_{1,1,-2,2}+4 S_{1,2,-2,1}+16 S_{2,-2,1,1}+ \\
& +4 S_{2,1,-2,1}-24 S_{1,-2,1,1,1}-16 S_{1,1,-2,1,1}
\end{aligned}
$$

\subsection{Parity invariance}

As we have seen in Section 2, the conserved charge $\mathfrak{Q}_{2}$ acquires perturbative corrections which shift the bare total conformal spin of Wilson operators by their anomalous dimension $\gamma(g)$ to the renormalized one,

$$
j_{0}=M+1 \quad \rightarrow \quad j=M+1+\frac{1}{2} \gamma(g) .
$$

This phenomenon implies that the anomalous dimensions can be defined more naturally as functions of the renormalized rather than bare spin, such that one can define a new function $P$ of argument $J$,

$$
\gamma=P\left(M+\frac{1}{2} \gamma\right)
$$


The parity invariance property of anomalous dimensions is then formulated as invariance of $P(j)$ under the reflection map $J \rightarrow-J$ with $J^{2}=j_{0}\left(j_{0}+1\right)$ [29, 30]. This condition results in an infinite number of relations for coefficients accompanying odd powers of the Lorentz spin in the large- $M$ expansion in terms of corresponding even powers. For finite $M$ this property gets translated into the presence of parity-even combinations of nested harmonic sums $\Omega_{k_{1}, k_{2}, \ldots}$ only, which can have positive even and negative odd $k_{i}$ 's [31, 32]. These functions are defined as follows. For a single-index harmonic sum, they do coincide with usual harmonic numbers, while for more than one index, they are defined recursively,

$$
\Omega_{k_{1}}=S_{k_{1}}, \quad \Omega_{k_{1}, k_{2}}=\omega_{k_{1}}\left(\Omega_{k_{2}}\right), \quad \Omega_{k_{1}, k_{2}, k_{3}}=\omega_{k_{1}}\left(\Omega_{k_{2}, k_{3}}\right), \ldots,
$$

with the involved map defined by

$$
\omega_{k_{1}}\left(S_{k_{2}, k_{3}, \ldots}\right)=S_{k_{1}, k_{2}, k_{3}, \ldots}-\frac{1}{2} S_{\operatorname{sign}\left(k_{1}\right) \operatorname{sign}\left(k_{2}\right)\left(\left|k_{1}\right|+\left|k_{2}\right|\right), k_{3} \ldots} .
$$

Let us establish the parity invariance property for the dressing part of the five-loop twist-two anomalous dimensions we determined above. Expanding Eq. (7.13) in the 't Hooft coupling and taking into account that dressing appears firstly at four loops, we find the five loop dressing contribution to $P(M)$

$$
\widehat{P}_{5}=\widehat{\gamma}_{5}-\frac{1}{4} \widehat{\gamma}_{4} \gamma_{1}^{\prime}-\frac{1}{2} \widehat{\gamma}_{4}^{\prime} \gamma_{1}
$$

where the hat on symbols denotes that we consider only their dressing parts. Explicitly, we use

$$
\begin{aligned}
& \gamma_{1}=2 S_{1}, \\
& \widehat{\gamma}_{4}=S_{1}\left(S_{-3}-S_{3}-2 S_{-2,1}\right) \zeta_{3}, \\
& \widehat{\gamma}_{5}=\zeta_{3} \gamma_{5}^{\zeta_{3}}+\zeta_{5} \gamma_{5}^{\zeta_{5}} .
\end{aligned}
$$

A long but straightforward calculation gives

$$
\widehat{P}_{5}=\zeta_{5} \widehat{P}_{5}^{\zeta_{5}}+\zeta_{3} \widehat{P}_{5}^{\zeta_{3}}
$$

where

$$
\begin{aligned}
\widehat{P}_{5}^{\zeta_{5}}= & -\frac{5}{4}\left(\Omega_{-4}-2 \Omega_{1,3}-2 \Omega_{3,1}-8 \Omega_{-2,1,1}-4 \Omega_{1,-2,1}\right) \\
\widehat{P}_{5}^{\zeta_{3}}= & -\frac{5}{2} \Omega_{-6}-2 \Omega_{-4,-2}-2 \Omega_{-2,-4}+\Omega_{3,3}+ \\
& +2\left(4 \Omega_{-4,1,1}+\Omega_{-2,1,3}+\Omega_{-2,3,1}+4 \Omega_{1,-4,1}+2 \Omega_{1,-2,3}+3 \Omega_{1,1,-4}+\Omega_{1,3,-2}+\right. \\
& \left.+\Omega_{3,-2,1}+\Omega_{3,1,-2}\right)+4\left(\Omega_{-2,-2,1,1}+2 \Omega_{-2,1,-2,1}+3 \Omega_{-2,1,1,-2}+2 \Omega_{1,-2,-2,1}+\right. \\
& \left.+2 \Omega_{1,-2,1,-2}+\Omega_{1,1,-2,-2}\right)-8\left(3 \Omega_{1,-2,1,1,1}+2 \Omega_{1,1,-2,1,1}\right)+ \\
& +2 \zeta_{2}\left(-\Omega_{-4}+\left(\Omega_{1,3}+\Omega_{3,1}\right)+2\left(3 \Omega_{-2,1,1}+2 \Omega_{1,-2,1}+\Omega_{1,1,-2}\right)\right)+ \\
& +7 \zeta_{4} \Omega_{1,1}
\end{aligned}
$$

which indeed obeys the parity-invariance properties spelled out at the beginning of the section. 


\section{Conclusions}

In this work we have developed an improved formalism for the analytical solution of the multiloop Baxter equation. As a demonstration of the efficiency of the framework, we found the four-loop Baxter polynomial and derived in a completely analytical form the resulting anomalous dimensions. This was possible largely due to an improved treatment of superficially non-polynomial terms in the Baxter equation. While in the previous consideration, the latter yielded multiple sums with Stirling numbers of the first and second kind involved and, as a consequence, hampering straightforward analytical calculation of derivatives of the Baxter function at fixed points which enter the definition of anomalous dimensions. They were treated making use of the principle of maximal transcendentality [20] by writing down the most general expression in terms of nested harmonic sums and then fitting the multiplicative rational coefficients to numerical data. In the current analysis this difficulty was overcome.

Next we found a more concise representation of the polynomial contribution to Baxter function by using the basis of Wilson rather than continuous Hahn polynomials. The complexity of these expressions was reduced roughly in half. Nevertheless it should be stated that our analysis of the non-polynomial terms is still in favor of a representation in continuous Hahn polynomials. It might still be interesting to completely restrict also the non-polynomial terms to a Wilson basis. As such it would be possible to compare oneto-one the analytic properties of the Baxter function of twist-two and -three operators, in order to pin down the origin of the asymptotic character of the Baxter equation as well as the Bethe ansatz.

Finally, we provided further evidence towards parity invariance of twist-two multiloop anomalous dimensions by calculating the dressing contribution to the five-loop result and showing that they obey the same theorem as was established earlier.

What we did not address are the wrapping effects in twist-two operators, which emerge starting from four loops. The latter are known to be described by a generalized Lüscher formula which reads for the case of twist- $L$ operators [21],

$$
\gamma^{(\text {wrap })}(g)=-i g^{4} \gamma_{0}^{2} \sum_{n=1}^{\infty} \operatorname{res}_{z=i n}\left(\frac{g^{2}}{z^{2}+n^{2}}\right)^{L} \frac{T^{2}(z, n)}{R(z, n)}+\mathcal{O}\left(g^{2(L+3)}\right) .
$$

It is written in terms of

$$
R(z, n)=Q_{0}\left(\frac{1}{2} z-\frac{i}{2}(n-1)\right) Q_{0}\left(\frac{1}{2} z+\frac{i}{2}(n-1)\right) Q_{0}\left(\frac{1}{2} z+\frac{i}{2}(n+1)\right) Q_{0}\left(\frac{1}{2} z-\frac{i}{2}(n+1)\right),
$$

and the function

$$
T(z, n)=\sum_{m=0}^{n-1} \frac{Q_{0}\left(\frac{1}{2} z-\frac{i}{2}(n-1)+i m\right)}{\left[\left(m-\frac{1}{2} n\right)-\frac{i}{2} z\right]\left[\left(m+1-\frac{1}{2} n\right)-\frac{i}{2} z\right]} .
$$

These correspond to one-loop corrections in a sigma model. The explicit formula for twisttwo operators was found in Ref. [21] and reads

$$
\gamma_{3}^{(\text {wrap })}=S_{1}^{2}\left(-5 \zeta_{5}-4 S_{-2} \zeta_{3}-2 S_{5}+2 S_{-5}+4 S_{4,1}-4 S_{3,-2}+4 S_{-2,-3}-8 S_{-2,-2,1}\right) .
$$


Our method can be viewed as a first step towards the analytical computation of the five-loop twist-two anomalous dimensions. Wrapping contributions are not included here. However the knowledge of the asymptotic prediction derived with it will allow one to analyze its analytical structure in the complex spin $M$ plane and constrain potential wrapping structures. These questions will be addressed elsewhere.

This work was supported by the U.S. National Science Foundation under grant no. PHY0757394 (A.B.) and by the Russian Foundation for Basic Research through Grant No. 0702-00902-a (A.K.). S.Z. thanks the DAAD for a short term lectureship and the University of Herat in Afghanistan for kind hospitality. S.Z. also thanks Matthias Staudacher for discussions. A.K. thanks the Albert-Einstein-Institut for kind hospitality and for a financial support.

\section{A Inhomogeneities}

The inhomogeneities of the four-loop expansion of the Baxter equation appear with the following multiplicative functions

$$
\begin{aligned}
\alpha^{+} & =\frac{1}{4} \frac{Q_{0}^{\prime \prime}\left(\frac{i}{2}\right)}{Q_{0}\left(\frac{i}{2}\right)}, \\
\beta^{+} & =\frac{i}{192} \frac{Q_{0}^{\prime \prime \prime}\left(\frac{i}{2}\right)}{Q_{0}\left(\frac{i}{2}\right)}, \\
\chi^{+} & =-\frac{1}{16} \frac{Q_{1}\left(\frac{i}{2}\right) Q_{0}^{\prime \prime}\left(\frac{i}{2}\right)}{Q_{0}^{2}\left(\frac{i}{2}\right)}+\frac{1}{16} \frac{Q_{1}^{\prime \prime}\left(\frac{i}{2}\right)}{Q_{0}\left(\frac{i}{2}\right)}+\frac{1}{192} \frac{Q_{0}^{(4)}\left(\frac{i}{2}\right)}{Q_{0}\left(\frac{i}{2}\right)}, \\
\delta^{+} & =\frac{Q_{1}\left(\frac{i}{2}\right)}{Q_{0}\left(\frac{i}{2}\right)}, \\
\varepsilon^{+} & =i \frac{Q_{1}^{\prime}\left(\frac{i}{2}\right)}{Q_{0}\left(\frac{i}{2}\right)},
\end{aligned}
$$

and analogous expressions whose argument has reverse sign being related to the above via

$$
\begin{aligned}
\alpha^{-}=\left(\alpha^{+}\right)^{*}= & \alpha^{+} \equiv \alpha, \quad \beta^{-}=-\left(\beta^{+}\right)^{*}=-\beta^{+}=-\beta, \\
\chi^{-}=\left(\chi^{+}\right)^{*}= & \chi^{+} \equiv \chi, \quad \delta^{-}=\left(\delta^{+}\right)^{*}=\delta^{+} \equiv \delta, \\
& \varepsilon^{-}=-\left(\varepsilon^{+}\right)^{*}=-\varepsilon^{+}=-\varepsilon .
\end{aligned}
$$

They can be written in terms of harmonic sums making use of the explicit solution to the Baxter equation and read

$$
\begin{aligned}
& \alpha=-S_{1}^{2}+S_{-2}, \\
& \beta=-\frac{1}{24}\left(S_{1}^{3}-S_{3}+3 S_{1,-2}-3 S_{-2,1}\right) \\
& \chi=\frac{1}{2}\left(S_{-4}-S_{4}-S_{-3,1}+S_{-2,-2}-S_{-2,2}-S_{1,-3}+S_{1,3}-S_{2,-2}+S_{2,2}+S_{3,1}\right)
\end{aligned}
$$




$$
\begin{aligned}
& +S_{-2,1,1}+S_{1,1,-2}-S_{1,1,2}-S_{1,2,1}-S_{2,1,1}+2 S_{1,1,1,1} \\
& +\frac{1}{4} \gamma_{0}\left(S_{-3}-S_{3}-S_{-2,1}+S_{1,-2}+S_{1,2}+S_{2,1}\right) \\
\delta= & a_{1} \\
\varepsilon= & -\gamma_{0}\left(S_{-2}+S_{2}\right)+2\left(S_{-3}-S_{3}-2 S_{-2,1}\right)+2 a_{1} S_{1},
\end{aligned}
$$

expressed in terms of nested harmonic sums

$$
S_{a_{1}, \ldots, a_{k}} \equiv S_{a_{1}, \ldots, a_{k}}(M)=\sum_{\ell_{1}=0}^{M} \frac{(-1)^{\operatorname{sign}\left(a_{1}\right)}}{\ell_{1}^{a_{1}}} \sum_{\ell_{2}=0}^{\ell_{1}} \frac{(-1)^{\operatorname{sign}\left(a_{2}\right)}}{\ell_{2}^{a_{2}}} \cdots \sum_{\ell_{k}=0}^{\ell_{k-1}} \frac{(-1)^{\operatorname{sign}\left(a_{k}\right)}}{\ell_{k}^{a_{k}}}
$$

\section{B Continuous Hahn and Wilson polynomials}

Continuous Hahn polynomials are defined as 33.

$$
p_{n}(u, a, b, c, d)=i^{n} \frac{(a+c)_{n}(a+d)_{n}}{n !}{ }_{3} F_{2}\left(\begin{array}{c}
-n, n+a+b+c+d-1, a+i u \\
a+c, a+d
\end{array} \mid 1\right) .
$$

They obey the functional relation $\left(P(u) \equiv p_{n}(\cdots)\right)$

$$
\begin{aligned}
& (c-i u)(d-i u) P(u+i)+(a+i u)(b+i u) P(u-i)+ \\
& \left(2 u^{2}-i(a+b-c-d) u-n^{2}+(-a-b-c-d+1) n-a b-c d\right) P(u)=0
\end{aligned}
$$

or

$$
\begin{gathered}
(c-i u)(d-i u)[P(u+i)-P(u)]+(a+i u)(b+i u)[P(u-i)-P(u)]+ \\
-\left(n^{2}+n(a+b+c+d-1) P(u)=0 .\right.
\end{gathered}
$$

Wilson polynomials are defined as

$$
\begin{aligned}
W_{n}\left(u^{2}, a, b, c, d\right)= & (a+b)_{n}(a+c)_{n}(a+d)_{n} \\
& \times{ }_{4} F_{3}\left(\begin{array}{c}
-n, n+a+b+c+d-1, a+i u, a-i u \\
a+b, a+c, a+d
\end{array}\right) .
\end{aligned}
$$

They obey the functional relation $\left(P(u) \equiv W_{n}\left(u^{2}, \cdots\right)\right)$

$$
\begin{aligned}
& (c-i u)(i a+u)(i b+u)(i d+u)(2 u-i) P(u+i) \\
& -(c+i u)(u-i a)(u-i b)(u-i d)(2 u+i) P(u-i)+2 i u A P(u)=0,
\end{aligned}
$$

with

$$
\begin{gathered}
A=2 u^{4}+u^{2}\left(-4 n^{2}-4(a+b+c+d-1) n+a+b+c+d+\right. \\
-2 a b-2 a c-2 b c-2 a d-2 b d-2 c d)+ \\
-n^{2}+(-a-b-c-d+1) n-a b c-a b d-a c d+2 a b c d-b c d .
\end{gathered}
$$


It can also be rewritten as

$$
n(n+s-1) Y(u)=B(u)[Y(u+i)-Y(u)]+B(-u)[Y(u-i)-Y(u)]
$$

with

$$
B(u)=-\frac{(u+i a)(u+i b)(u+i c)(u+i d)}{2 u(2 u+i)} .
$$

The relation

$$
p_{M}\left(u, \frac{1}{2}, \frac{1}{2}, \frac{1}{2}, \frac{1}{2}\right)=\text { constant } \times W_{M / 2}\left(u, \frac{1}{2}, \frac{1}{2}, \frac{1}{2}, 0\right),
$$

is immediately proved since the two recurrence relation are equal for the above choice of parameters. Looking at the normalization, one can check that the constant is indeed equal to one.

The comparison of the two-loop Baxter equation and equations obeyed by polynomials yield the identifications for the continuous Hahn

$$
n=M, \quad a=c=\frac{1}{2}+\frac{i}{\sqrt{2}} g+\frac{1}{4} g^{2} \gamma_{0}, \quad b=d=\frac{1}{2}-\frac{i}{\sqrt{2}} g+\frac{1}{4} g^{2} \gamma_{0},
$$

and Wilson polynomials,

$$
\begin{aligned}
& n=\frac{1}{2} M, \quad s=\frac{3}{2}+\frac{1}{2} g^{2} \gamma_{0}, \quad a=\frac{1}{2}, \quad d=0, \\
& b=\frac{1}{2}+\frac{i}{\sqrt{2}} g+\frac{1}{4} g^{2} \gamma_{0}, \quad c=\frac{1}{2}-\frac{i}{\sqrt{2}} g+\frac{1}{4} g^{2} \gamma_{0},
\end{aligned}
$$

respectively.

\section{Derivatives of the Baxter functions}

The $n$-th derivative of $Q^{(0)}(u)$ evaluated at $u=i / 2$ is a combination of harmonic sums with uniform transcendentality $n$ and multi-indices containing only 1 and \pm 2 . The first cases are

$$
\begin{aligned}
Q_{0}^{\prime}\left(\frac{i}{2}\right)= & -2 i S_{1} \\
Q_{0}^{\prime \prime}\left(\frac{i}{2}\right)= & -4\left(S_{-2}-S_{2}+2 S_{1,1}\right) \\
Q_{0}^{\prime \prime \prime}\left(\frac{i}{2}\right)= & -24 i\left(S_{-2,1}-S_{1,-2}+S_{1,2}+S_{2,1}-2 S_{1,1,1}\right) \\
Q_{0}^{(4)}\left(\frac{i}{2}\right)= & 96\left(S_{-2,-2}-S_{-2,2}-S_{2,-2}+S_{2,2}+2 S_{-2,1,1}-2 S_{1,-2,1}+2 S_{1,1,-2}+\right. \\
& \left.-2 S_{1,1,2}-2 S_{1,2,1}-2 S_{2,1,1}+4 S_{1,1,1,1}\right) \\
Q_{0}^{(5)}\left(\frac{i}{2}\right)= & -960 i\left(S_{-2,-2,1}-S_{-2,1,-2}+S_{-2,1,2}+S_{-2,2,1}+S_{1,-2,-2}-S_{1,-2,2}+\right. \\
& -S_{1,2,-2}+S_{1,2,2}+S_{2,-2,1}-S_{2,1,-2}+S_{2,1,2}+S_{2,2,1}-2 S_{-2,1,1,1}+ \\
& 2 S_{1,-2,1,1}-2 S_{1,1,-2,1}+2 S_{1,1,1,-2}-2 S_{1,1,1,2}-2 S_{1,1,2,1}-2 S_{1,2,1,1}+ \\
& \left.-2 S_{2,1,1,1}+4 S_{1,1,1,1,1}\right) \\
Q_{0}^{(6)}\left(\frac{i}{2}\right)= & -5760\left(S_{-2,-2,-2}-S_{-2,-2,2}-S_{-2,2,-2}+S_{-2,2,2}-S_{2,-2,-2}+\right.
\end{aligned}
$$




$$
\begin{aligned}
& +S_{2,-2,2}+S_{2,2,-2}-S_{2,2,2}+2 S_{-2,-2,1,1}-2 S_{-2,1,-2,1}+2 S_{-2,1,1,-2}+ \\
& -2 S_{-2,1,1,2}-2 S_{-2,1,2,1}-2 S_{-2,2,1,1}+2 S_{1,-2,-2,1}-2 S_{1,-2,1,-2}+ \\
& +2 S_{1,-2,1,2}+2 S_{1,-2,2,1}+2 S_{1,1,-2,-2}-2 S_{1,1,-2,2}-2 S_{1,1,2,-2}+2 S_{1,1,2,2}+ \\
& +2 S_{1,2,-2,1}-2 S_{1,2,1,-2}+2 S_{1,2,1,2}+2 S_{1,2,2,1}-2 S_{2,-2,1,1}+2 S_{2,1,-2,1}+ \\
& -2 S_{2,1,1,-2}+2 S_{2,1,1,2}++2 S_{2,1,2,1}+2 S_{2,2,1,1}+4 S_{-2,1,1,1,1}-4 S_{1,-2,1,1,1}+ \\
& 4 S_{1,1,-2,1,1}-4 S_{1,1,1,-2,1}+4 S_{1,1,1,1,-2}-4 S_{1,1,1,1,2}-4 S_{1,1,1,2,1}-4 S_{1,1,2,1,1}+ \\
& -4 S_{1,2,1,1,1}-4 S_{2,1,1,1,1}+8 S_{1,1,1,1,1,1} .
\end{aligned}
$$

Let us write the two-loop Baxter polynomial as

$$
Q_{1}(u)=a_{1}(M) Q_{0}(M)+\delta Q_{1}(u),
$$

where $a_{1}$ is the same as in Eq. (3.8). The derivatives of $Q^{(1)}(u)$ are expressed in terms of the derivatives of $Q^{(0)}(u)$ and those of $\delta Q^{(1)}(u)$. The $n$-th derivative of $\delta Q^{(1)}(u)$ can be expressed in terms of harmonic sums with uniform transcendentality $n+2$. The first cases are

$$
\begin{aligned}
\delta Q_{1}\left(\frac{i}{2}\right)= & 0 \\
\delta Q_{1}^{\prime}\left(\frac{i}{2}\right)= & -2 i\left(2 S_{-3}-3 S_{-2,1}-S_{1,-2}-S_{1,2}-S_{2,1}\right) \\
\delta Q_{1}^{\prime \prime}\left(\frac{i}{2}\right)= & 8\left(S_{-3,1}+S_{-2,2}-S_{1,-3}-S_{1,3}-S_{2,-2}-2 S_{2,2}-S_{3,1}+\right. \\
& \left.-2 S_{-2,1,1}+2 S_{1,-2,1}+2 S_{1,1,-2}+2 S_{1,1,2}+2 S_{1,2,1}+2 S_{2,1,1}\right) \\
\delta Q_{1}^{\prime \prime \prime}\left(\frac{i}{2}\right)= & -24 i\left(2 S_{-4,1}-S_{-3,-2}+5 S_{-3,2}+S_{-2,-3}+S_{-2,3}-S_{2,-3}+\right. \\
& +3 S_{2,3}+S_{3,-2}+3 S_{3,2}+2 S_{4,1}-8 S_{-3,1,1}-4 S_{-2,-2,1}+2 S_{-2,1,-2}+ \\
& -6 S_{-2,1,2}-6 S_{-2,2,1}-4 S_{1,1,3}-2 S_{1,2,-2}-6 S_{1,2,2}-4 S_{1,3,1}-2 S_{2,1,-2}+ \\
& -6 S_{2,1,2}-6 S_{2,2,1}-4 S_{3,1,1}+10 S_{-2,1,1,1}-2 S_{1,-2,1,1}+2 S_{1,1,-2,1}+ \\
& \left.+6 S_{1,1,1,-2}+6 S_{1,1,1,2}+6 S_{1,1,2,1}+6 S_{1,2,1,1}+6 S_{2,1,1,1}\right) \\
\delta Q_{1}^{(4)}\left(\frac{i}{2}\right)= & 384\left(S_{-3,-3}-S_{-3,3}-S_{3,-3}+S_{3,3}-S_{-3,-2,1}+2 S_{-3,1,2}+\right. \\
& +2 S_{-3,2,1}+S_{-2,1,3}+2 S_{-2,2,2}+S_{-2,3,1}-S_{1,-4,1}-2 S_{1,-3,2}+ \\
& -S_{1,-2,-3}+S_{1,2,-3}-2 S_{1,2,3}-2 S_{1,3,2}-S_{1,4,1}-S_{2,-3,1}-S_{2,-2,2}+ \\
& +S_{2,1,-3}-2 S_{2,1,3}-3 S_{2,2,2}-2 S_{2,3,1}+S_{3,-2,1}-2 S_{3,1,2}-2 S_{3,2,1}+ \\
& -3 S_{-3,1,1,1}-S_{-2,1,-2,1}-3 S_{-2,1,1,2}-3 S_{-2,1,2,1}-3 S_{-2,2,1,1}+3 S_{1,-3,1,1}+ \\
& +2 S_{1,-2,-2,1}-S_{1,-2,1,-2}+2 S_{1,-2,1,2}+2 S_{1,-2,2,1}+S_{1,1,-3,1}+S_{1,1,-2,2}+ \\
& -S_{1,1,1,-3}+3 S_{1,1,1,3}+S_{1,1,2,-2}+4 S_{1,1,2,2}+3 S_{1,1,3,1}+S_{1,2,1,-2}+ \\
& +4 S_{1,2,1,2}+4 S_{1,2,2,1}+3 S_{1,3,1,1}+S_{2,-2,1,1}+S_{2,1,1,-2}+4 S_{2,1,1,2}+ \\
& +4 S_{2,1,2,1}+4 S_{2,2,1,1}+3 S_{3,1,1,1}+4 S_{-2,1,1,1,1}-4 S_{1,-2,1,1,1}+ \\
& \left.-4 S_{1,1,1,1,-2}-4 S_{1,1,1,1,2}-4 S_{1,1,1,2,1}-4 S_{1,1,2,1,1}-4 S_{1,2,1,1,1}-4 S_{2,1,1,1,1}\right)
\end{aligned}
$$




\section{Mellin transformation}

In this appendix we devise a very efficient formalism for finding solutions to the secondorder finite difference Baxter equation focusing on non-polynomial inhomogeneities. The results that we will present are very general, covering all possible powers of non-polynomial contributions, $\left(u^{ \pm}\right)^{-k} Q_{\ell}(u)$, and are therefore applicable to any order of perturbation theory. The restriction and application of the machinery to three- and four-loop non- polynomial inhomogeneities, which are the main objective of the current study, are given in sections 5.1 and 5.2, respectively. We also employ it to the dressing-induced five-loop non-polynomial part in section 7. Below, we first introduce the Mellin transform for the Baxter function and accompanying (inverse) polynomial dressing factors in the spectral parameter. Then we perform a variable transformation which is particularly convenient for carrying out Mellin convolutions and inverse transform.

To start with let us introduce the Mellin transform of the main ingredients. For the Baxter function $Q(u)$, where the loop-order subscript is dropped being irrelevant, it takes the form [28]

$$
Q(u)=K \int_{0}^{\infty} d \omega \omega^{i u-1} Q(-\omega), \quad K=\frac{1}{\Gamma(i u) \Gamma(1-i u)} .
$$

While for the function with the argument shifted by $i$, i.e., $Q(u \pm i)$, we find that in Mellin space this yields a multiplication by a power of $\omega$

$$
Q(u \pm i)=-K \int_{0}^{\infty} d \omega \omega^{i u-1}\left\{\omega^{\mp 1} Q(-\omega)\right\}=K \int_{0}^{\infty} d \omega \omega^{i u-1}\left\{-\omega^{\mp 1} Q(-\omega)\right\} .
$$

Next, turning to terms where $Q(u)$ is accompanied by a positive power of a polynomial in the spectral parameter of the form $(u+b i)^{L} \equiv \lambda^{L}$, we get at first

$$
(u+b i)^{L} Q(u)=\lambda^{L} K \int_{0}^{\infty} d \omega \omega^{i \lambda-1} \omega^{b} Q(-\omega) .
$$

Then, we can re-express the product $\lambda^{L} \omega^{i \lambda-1}$ by a differential operator acting on the exponent, i.e., $-i(d / d \omega \omega)^{L} \omega^{i \lambda-1}$, and subsequently integrate by parts to find

$$
(u+b i)^{L} Q(u)=K \int_{0}^{\infty} d \omega \omega^{i u-1} \omega^{-b}\left[i \omega \frac{d}{d \omega}\right]^{L}\left\{\omega^{b} Q(-\omega)\right\} .
$$

Similarly, for the Baxter function with the shifted argument $Q(u \pm i)$, we get the expression

$$
(u+b i)^{L} Q(u \pm i)=K \int_{0}^{\infty} d \omega \omega^{i u-1} \omega^{-b}\left[i \omega \frac{d}{d \omega}\right]^{L}\left\{-\omega^{b \mp 1} Q(-\omega)\right\} .
$$

In the same vein we can consider terms with inverse powers of the same polynomial, i.e., $(u+b i)^{-L}$. First, notice that for $L=1$, its Mellin integral representation

$$
\frac{1}{u+b i}= \pm i \int_{0}^{\infty} d \omega \omega^{i u-1-b} \theta( \pm 1 \mp \omega)=\left\{\begin{array}{l}
+i \int_{0}^{1} d \omega \omega^{i u-1-b} \\
-i \int_{1}^{\infty} d \omega \omega^{i u-1-b}
\end{array}, \quad \text { for } \quad b \lessgtr 0\right.
$$


is given in terms of the the Heaviside step function $\theta(x)$, defined conventionally as

$$
\theta(x)= \begin{cases}1, & \text { for } \quad x \geq 0 \\ 0, & \text { for } \quad x<0\end{cases}
$$

The other observation is the well-known fact that the Mellin transform of a product of functions is realized as a convolution of their Mellin transforms. Namely, if a function $M_{j}(u)$ is expressed in terms of its Mellin transform

$$
M_{j}(u)=\int_{0}^{\infty} d \omega \omega^{i u-1} F_{j}(\omega),
$$

then the product of $M_{1}(u) M_{2}(u)$ corresponds to

$$
M_{1}(u) M_{2}(u)=\int_{0}^{\infty} d \omega \omega^{i u-1} \int_{0}^{\infty} \frac{d \omega_{1}}{\omega_{1}} F_{1}\left(\omega_{1}\right) F_{2}\left(\frac{\omega}{\omega_{1}}\right)
$$

which we will write formally as

$$
M_{1}(u) M_{2}(u) \stackrel{M^{-1}}{\rightarrow} \int_{0}^{\infty} \frac{d \omega_{1}}{\omega_{1}} F_{1}\left(\omega_{1}\right) F_{2}\left(\frac{\omega}{\omega_{1}}\right)
$$

where the symbol $\stackrel{M^{-1}}{\rightarrow}$ implies the inverse Mellin transform. Putting the two results together, leads to

$$
\begin{aligned}
\frac{1}{u+b i} Q(u) \stackrel{M^{-1}}{\rightarrow} & \pm i K \int_{0}^{\infty} \frac{d \omega_{1}}{\omega_{1}} Q\left(-\omega_{1}\right)\left(\frac{\omega}{\omega_{1}}\right)^{-b} \theta\left( \pm 1 \mp \frac{\omega}{\omega_{1}}\right) \\
& =\left\{\begin{array}{l}
+i K \int_{\omega}^{\infty} \frac{d \omega_{1}}{\omega_{1}}\left(\frac{\omega}{\omega_{1}}\right)^{-b} Q\left(-\omega_{1}\right) \\
-i K \int_{0}^{\omega} \frac{d \omega_{1}}{\omega_{1}}\left(\frac{\omega}{\omega_{1}}\right)^{-b} Q\left(-\omega_{1}\right)
\end{array}, \text { for } \quad b \lessgtr 0 .\right.
\end{aligned}
$$

Now, upon differentiating both sides of this equation w.r.t. $b$, we obtain the final result

$$
\frac{L !}{(u+b i)^{L}} Q(u) \stackrel{M^{-1}}{\rightarrow}\left\{\begin{array}{l}
-(-i)^{L+1} K \int_{\omega}^{\infty} \frac{d \omega_{1}}{\omega_{1}}\left(\ln \frac{\omega}{\omega_{1}}\right)^{L}\left(\frac{\omega}{\omega_{1}}\right)^{-b} Q\left(-\omega_{1}\right) \\
+(-i)^{L+1} K \int_{0}^{\omega} \frac{d \omega_{1}}{\omega_{1}}\left(\ln \frac{\omega}{\omega_{1}}\right)^{L}\left(\frac{\omega}{\omega_{1}}\right)^{-b} Q\left(-\omega_{1}\right)
\end{array}, \quad \text { for } \quad b \lessgtr 0 .\right.
$$

And for the shifted Baxter polynomial $Q(u \pm i)$, this is replaced by

$$
\begin{aligned}
& \frac{L !}{(u+b i)^{L}} Q(u \pm i) \\
& \stackrel{M^{-1}}{\rightarrow}\left\{\begin{array}{l}
-(-i)^{L+1} K \int_{\omega}^{\infty} \frac{d \omega_{1}}{\omega_{1}}\left(\ln \frac{\omega}{\omega_{1}}\right)^{L}\left(\frac{\omega}{\omega_{1}}\right)^{-b}\left[-\omega_{1}^{\mp 1} Q\left(-\omega_{1}\right)\right] \\
+(-i)^{L+1} K \int_{0}^{\omega} \frac{d \omega_{1}}{\omega_{1}}\left(\ln \frac{\omega}{\omega_{1}}\right)^{L}\left(\frac{\omega}{\omega_{1}}\right)^{-b}\left[-\omega_{1}^{\mp 1} Q\left(-\omega_{1}\right)\right]
\end{array}, \quad \text { for } \quad b \lessgtr 0 .\right.
\end{aligned}
$$




\section{D.1 Change of variables}

To proceed further, we introduce the variable $z$ as in [28],

$$
\omega=\frac{z}{1-z},
$$

such that the Mellin transform (D.1) takes the form

$$
Q(u)=K_{1} \int_{0}^{1} d z z^{i u-1 / 2}(1-z)^{-i u-1 / 2} \Psi(z),
$$

where we introduced a new function (see Ref. [23])

$$
Q(z)=\sqrt{z(1-z)} \Psi(z),
$$

with the corresponding normalization factor

$$
K_{1}=\frac{1}{\Gamma\left(\frac{1}{2}-i u\right) \Gamma\left(\frac{1}{2}+i u\right)} .
$$

The latter leads to the unit normalization factor in Eq. (3.1).

Absorbing all terms of the all-order Baxter equation beyond one loop into inhomogeneities $\widehat{Q}(u)$ on the right-hand side, we can write it as

$$
\left(u^{+}\right)^{2} Q(u+i)+\left(u^{-}\right)^{2} Q(u-i)-t_{0}(u) Q(u)=\widehat{Q}(u) .
$$

While the corresponding equation in Mellin space reads (we omitted the overall factor $K_{1} \sqrt{z(1-z)}$ on both left- and right-hand sides)

$$
z(1-z) \Psi^{\prime \prime}(z)+(1-2 z) \Psi^{\prime}(z)+M(M+1) \Psi(z)=\widehat{\Psi}(z),
$$

and where the primes on $\Psi(z)$, e.g., $\Psi^{\prime}(z)$, correspond to the differentiation w.r.t. the variable $z$.

The functions $Q(u)$ and $\Psi(z)$ admit perturbative expansions

$$
Q(u)=\sum_{\ell=0}^{\infty} g^{2 \ell} Q_{\ell}(u), \quad \Psi(z)=\sum_{\ell=0}^{\infty} g^{2 \ell} \Psi_{\ell}(z),
$$

with their coefficients taking the form

$$
Q_{\ell}(u)=\sum_{k=0}^{M} R_{\ell, k}(M) P_{k}(u), \quad \Psi_{\ell}(z)=\sum_{k=0}^{M} R_{\ell, k}(M) z^{k} .
$$

In the following, we will reserve the convention $R_{0, k}(M)=R_{k}(M)$ for the leading order term, with the functions $R_{k}(M)$ and $P_{k}(u)$ introduced in Eq. (5.3). 
Note that the solution of (D.19) can be written as a sum of the $z^{k}$ and $(1-z)^{k}$ expansions, i.e.,

$$
\Psi_{\ell}(z)=\frac{1}{2} \sum_{k=0}^{M}\left[z^{k}+(1-z)^{k}\right] R_{\ell, k}(M),
$$

which corresponds to the real part of its Mellin transform counterpart (D.21), i.e.,

$$
Q_{\ell}(u)=\sum_{k=0}^{M} \Re \mathrm{e}\left[P_{k}(u)\right] R_{\ell, k}(M)
$$

and is the solution that is sought for.

\section{D.2 General properties}

Consider the polynomial solution (D.21) to the Baxter equation (D.19). The left-hand side of the latter can be cast in the form

$$
\sum_{k=0}^{M-1}\left[(k+1)^{2} R_{\ell, k+1}(M)-(M-k)(k+1+M) R_{\ell, k}(M)\right] z^{k}
$$

and contains the maximal $(M-1)$-th power of the $z$-variable compared to the solution (D.21) which is a $M$-th order polynomial.

It is convenient to introduce the new coefficients $r_{\ell, k+1}(M)$ as

$$
r_{\ell, k}(M)=\frac{R_{\ell, k}(M)}{R_{k}(M)}
$$

with $R_{k}(M)$ given in Eq. (5.3) . Then, since it obeys the recurrence relation

$$
R_{k}(M)=R_{k+1}(M) \frac{(k+1)^{2}}{(M-k)(k+1+M)},
$$

we can replace (D.24) by

$$
\sum_{k=0}^{M-1} z^{k} R_{k}(M)(k+1)^{2}\left[r_{\ell, k+1}(M)-r_{\ell, k}(M)\right]
$$

At leading order of perturbation theory, the right-hand side $\widehat{\Psi}(z)$ of Eq. (D.19) vanishes, and the equation for $r_{\ell, k}$ is simply

$$
r_{\ell, k+1}(M)-r_{\ell, k}(M)=0
$$

The solution is

$$
r_{\ell, k}(M)=r_{\ell, 0}(M), \quad R_{0, k}(M)=r_{\ell, 0}(M) \cdot R_{k}(M),
$$


where $r_{\ell, 0}(M)$ is a constant. The possible presence of nontrivial contributions from the $(1-z)^{k}$ part of the expansion in the right-hand side of Eq. (D.19) does not alter any of the above results because its left-hand side is symmetric under the exchange $z \rightarrow(1-z)$.

Note that in order to have the solution of Eq. (D.19) in the form of (D.21), the inhomogeneities $\widehat{Q}(u)$ and $\widehat{\Psi}(z)$ should be expanded in the same basis of functions

$$
\widehat{Q}(u)=\sum_{k=0}^{M-1} P_{k}(u) B_{k+1}, \quad \widehat{\Psi}(z)=\sum_{k=0}^{M-1} B_{k+1} z^{k},
$$

and, respectively,

$$
\widehat{Q}(u)=\sum_{k=0}^{M-1} \Re \mathrm{e}\left[P_{k}(u)\right] B_{k+1}, \quad \widehat{\Psi}(z)=\frac{1}{2} \sum_{k=0}^{M}\left[z^{k}+(1-z)^{k}\right] B_{k+1},
$$

if the $(1-z)^{k}$-expansion constitutes a nontrivial contribution.

Going beyond leading order of perturbation theory, the inhomogeneities in the Baxter equation affect the recurrence relation for the expansion coefficients (D.25) as follows

$$
r_{\ell, k+1}(M)-r_{\ell, k}(M)=\frac{B_{k+1}}{R_{k+1}(M)(k+1)^{2}},
$$

such that the iterative solution to it reads

$$
r_{\ell, k}(M)=r_{\ell, 0}(M)+\sum_{l=1}^{k} \frac{B_{l}}{R_{l}(M) l^{2}} .
$$

Here, the first term in (D.33) corresponds to the general solution of the homogeneous equation and the second one is a particular solution of the inhomogeneous equation (D.19).

\section{D.3 Polynomial inhomogeneities}

Starting from two-loop order, the right-hand side of Eq. (D.18) is nonzero and contains power-series contribution in $\sim\left(u^{ \pm}\right)^{k}$ with $k \leq 1$. The terms $\sim u^{1}$ and $\sim u^{0}$ induce polynomial contributions. The bulk of them can be calculated directly in the $u$-space (see Section 4). However, it is instructive to consider their calculation also in the Mellin transform $z$-space, because, starting from 4-loop, nonpolynomial effects from lower orders of perturbation theory will re-emerge through these terms as well.

For simplicity and since this is all one needs for the present analysis, we limit our consideration to contributions at $n$-th order of perturbation theory from Baxter functions of one loop order lower. All other cases can be treated similarly since the only difference will be in the coefficients accompanying $u^{1}$ and $u^{0}$. 


\section{D.3.1 Contributions $\sim u^{1}$}

For the case at hand, the corresponding inhomogeneity in the right-hand side has the following form (see also Eq. (5.48))

$$
i u^{+} \widehat{Q}_{1}(u+i)-i u^{-} \widehat{Q}_{1}(u-i)+(2 M+1) \widehat{Q}_{1}(u),
$$

with a function $\widehat{Q}_{1}(u)$. After the Mellin transformation to $z$-space (similar to Eq. (D.15)) and cancelling the overall coefficient $K_{1} \sqrt{z(1-z)}$, we find

$$
(1-2 z) \widehat{\Psi}_{1}^{\prime}(z)+2 M \widehat{\Psi}_{1}(z) .
$$

Let now $\widehat{Q}_{1}(u)$ and $\widehat{\Psi}_{1}(z)$ have the following expansions (analogous to (D.22) and (D.23) $)$

$$
\widehat{Q}_{1}(u)=\sum_{k=0}^{M} \Re \mathrm{e}\left[P_{k}(u)\right] \widehat{F}_{1}(k), \quad \widehat{\Psi}_{1}(z)=\frac{1}{2} \sum_{k=0}^{M}\left[z^{k}+(1-z)^{k}\right] \widehat{F}_{1}(k) .
$$

Then, the corresponding particular solutions, $\bar{Q}_{1}(u)$ and $\bar{\Psi}_{1}(z)$, of the Baxter equation and its Mellin analogue, can be found in terms of the expansion coefficients $\widehat{F}_{1}(k)$

$$
\bar{Q}_{1}(u)=\sum_{k=0}^{M} \Re \mathrm{e}\left[P_{k}(u)\right] F_{1}(k), \quad \bar{\Psi}_{1}(z)=\frac{1}{2} \sum_{k=0}^{M}\left[z^{k}+(1-z)^{k}\right] F_{1}(k),
$$

where the coefficients $F_{1}(k)$ are determined by $\widehat{F}_{1}(k)$ by Eqs. (D.32) and (D.33).

It is possible to consider the expansions in $z^{k}$ and $(1-z)^{k}$ independently. Addressing the $z^{k}$-expansion first, we have from (D.35) and (D.36)

$$
\begin{aligned}
& \sum_{k=0}^{M-1} z^{k}\left[(k+1) \widehat{F}_{1}(k+1)+2(M-k) \widehat{F}_{1}(k)\right] \\
& =\sum_{k=0}^{M-1} z^{k} R_{k+1}(M)(k+1)^{2}\left[\frac{\widehat{f_{1}}(k+1)}{k+1}-\frac{2 \widehat{f}_{1}(k)}{k+1+M}\right],
\end{aligned}
$$

where we factored out the $R_{k}$ dependence and as a result introduced new coefficients

$$
F_{1}(k)=R_{k}(M) f_{1}(k), \quad \widehat{F}_{1}(k)=R_{k}(M) \widehat{f}_{1}(k) .
$$

Next, putting Eqs. (D.30), (D.32) and (D.38) together, we get for $f_{1}(k)$ the following representation

$$
f_{1}(k)=f_{1}(0)+\sum_{m=1}^{k}\left[\frac{\widehat{f_{1}}(m)}{m}-\frac{2 \widehat{f}_{1}(m-1)}{m+M}\right] .
$$

Finally, when we set $\widehat{Q}_{1}(u)=Q_{0}(u)$, i.e. to the leading order Baxter polynomial, the coefficients can be found explicitly and read

$$
\widehat{f}_{1}(k)=1, \quad f_{1}(k)=S_{1}(k)+2\left(S_{1}(k+M)-S_{1}(M)\right) .
$$

In this way we thus reproduce the $\gamma_{0}$-part of the $Q_{1}$ Baxter function in (5.30). 


\section{D.3.2 Contributions $\sim u^{0}$}

For the $u^{0}$ term, the corresponding right-hand side has the following form (see also Eq. $(5.48))$

$$
\widehat{Q}_{2}(u+i)+\widehat{Q}_{2}(u-i)-2 \widehat{Q}_{2}(u),
$$

in terms of a function $\widehat{Q}_{2}(u)$, which we will specify later. After the Mellin transform and again cancelling the overall coefficient $K_{1} \sqrt{z(1-z)}$, we find for the $z$-space counterpart of the above inhomogeneity

$$
\frac{1}{z(1-z)} \widehat{\Psi}_{2}(z) \text {. }
$$

Here $\widehat{Q}_{2}(u)$ and $\widehat{\Psi}_{2}(z)$ have the following expansions

$$
\widehat{Q}_{2}(u)=\sum_{k=1}^{M} \Re \mathrm{e}\left[P_{k}(u)\right] \widehat{F}_{2}(k), \quad \widehat{\Psi}_{2}(z)=\frac{1}{2} \sum_{k=1}^{M}\left[z^{k}+(1-z)^{k}\right] \widehat{F}_{2}(k) .
$$

As it was discussed in the previous section, the expansions (D.44) prevent the appearance of negative powers of $z$ and/or $(1-z)$ in the right-hand side of the Baxter equation. Then the corresponding particular solutions $\bar{Q}_{2}(u)$ and $\bar{\Psi}_{2}(z)$ to the Baxter equation can be found in a similar fashion

$$
\bar{Q}_{2}(u)=\sum_{k=0}^{M} \Re \mathrm{e}\left[P_{k}(u)\right] F_{2}(k), \quad \bar{\Psi}_{2}(z)=\frac{1}{2} \sum_{k=0}^{M}\left[z^{k}+(1-z)^{k}\right] F_{2}(k),
$$

where the coefficients $F_{2}(k)$ are determined by $\widehat{F}_{2}(k)$.

In the following, it is convenient to separately consider the cases of symmetric and non-symmetric functions $\widehat{Q}_{2}(u)$ and $\widehat{\Psi}_{2}(z)$ with respect to the replacements $u \rightarrow-u$ and $z \rightarrow(1-z)$, respectively.

Symmetric case. In this case, $\widehat{\Psi}_{2}(1-z)=\widehat{\Psi}_{2}(z)$ and Eq. (D.43) is equal to

$$
\frac{1}{z} \widehat{\Psi}_{2}(z)+\frac{1}{(1-z)} \widehat{\Psi}_{2}(z) \text {. }
$$

Considering only the $z^{k}$-part of the expansion, we find from (D.46)

$$
\sum_{k=0}^{M-1} z^{k} \widehat{F}_{2}(k+1)
$$

Then, introducing new coefficients via

$$
F_{2}(k)=R_{k}(M) f_{2}(k), \quad \widehat{F}_{2}(k)=R_{k}(M) \widehat{f}_{2}(k),
$$


we deduce from Eqs. (D.30), (D.32) and (D.47) the following representation

$$
f_{2}(k)=f_{2}(0)+2 \sum_{m=1}^{k} \frac{\widehat{f_{2}}(m)}{m^{2}} .
$$

If $\widehat{Q}_{2}(u)=Q_{0}$, the coefficients read

$$
\widehat{f}_{2}(k)=1, \quad f_{2}(k)=2 S_{2}(k),
$$

and, thus, they reproduce the $Q_{1}$ Baxter function, when $\gamma_{0}=\tilde{b}(M)=0$ (see (5.30)).

Non-symmetric case. As in the symmetric case, we can cast it in the form of the $z^{k}$ expansion,

$$
\frac{1}{z(1-z)} \sum_{k=1}^{M} z^{k} \widehat{F}_{2}(k)=\sum_{m=0}^{\infty} z^{m-1} \sum_{k=1}^{M} z^{k} \widehat{F}_{2}(k)=\sum_{k=1}^{M} z^{k} \widehat{F}_{2}(k) \sum_{p=k}^{\infty} z^{p-1},
$$

where $p=m+k$, however, now being an infinite series rather than a finite sum. Splitting the series $\sum_{p=k}^{\infty}$ into two parts

$$
\sum_{p=k}^{\infty}=\sum_{p=k}^{M}+\sum_{p=M+1}^{\infty}
$$

we replace the right-hand side of (D.51) by

$$
\sum_{p=1}^{M} z^{p-1} \widehat{\Phi}_{2}(p)+\sum_{p=M+1}^{\infty} z^{p-1} \widehat{\Phi}_{2}(M)
$$

where

$$
\widehat{\Phi}_{2}(p)=\sum_{k=1}^{p} \widehat{F}_{2}(k) .
$$

Then, Eq. (D.53) can be represented as

$$
\sum_{p=1}^{M} z^{p-1}\left[\widehat{\Phi}_{2}(p)-\widehat{\Phi}_{2}(M)\right]+\widehat{\Phi}_{2}(M) \sum_{p=1}^{\infty} z^{p-1}=\sum_{p=1}^{M} z^{p-1}\left[\widehat{\Phi}_{2}(p)-\widehat{\Phi}_{2}(M)\right]+\frac{\widehat{\Phi}_{2}(M)}{1-z} .
$$

As a consequence, Eq. (D.43) reads

$$
\frac{1}{z(1-z)} \widehat{\Psi}_{2}(z)=\frac{1}{2} \sum_{p=1}^{M}\left(z^{p-1}+(1-z)^{p-1}\right)\left[\widehat{\Phi}_{2}(p)-\widehat{\Phi}_{2}(M)\right]+\frac{\widehat{\Phi}_{2}(M)}{2 z(1-z)} .
$$


To proceed further we have to address the nonpolynomial term in Eq. (D.56). Notice that if we add to the left-hand side of (D.19) the Baxter equation obeyed by the solution $Q_{1}(u)$ with the formal condition $\gamma_{0}=0$, this will yield the following contribution to the inhomogeneity

$$
\frac{K_{2}}{z(1-z)} \Psi_{0}(z)
$$

where $\Psi_{0}(z)$ is the leading order solution of the Baxter equation with the property

$$
\Psi_{0}(z)=1+\widetilde{\Psi}_{0}(z), \quad \widetilde{\Psi}_{0}(z)=\sum_{k=1}^{M} z^{k} R_{k}(M) .
$$

Put together, we now have the right-hand side in the form

$$
\frac{1}{2} \sum_{p=1}^{M}\left(z^{p-1}+(1-z)^{p-1}\right)\left[\widehat{\Phi}_{2}(p)-\widehat{\Phi}_{2}(M)+2 K_{2} R_{k}(M)\right]+\left[\widehat{\Phi}_{2}(M)+2 K_{2}\right] \frac{1}{2 z(1-z)} .
$$

Choosing $K_{2}=-\widehat{\Phi}_{2}(M) / 2$, we cancel the unwanted term $\sim 1 /(z(1-z))$ and find that the resulting Baxter equation is obeyed by the function

$$
\bar{Q}_{2}(u)-\left.\frac{1}{2} \widehat{\Phi}_{2}(M) Q_{1}(u)\right|_{\gamma_{0}=0},
$$

which admits the following Mellin-space transform

$$
\frac{1}{2} \sum_{p=1}^{M}\left(z^{p-1}+(1-z)^{p-1}\right)\left[\widehat{\Phi}_{2}(p)-\widehat{\Phi}_{2}(M)\left(1+R_{p}(M)\right)\right] .
$$

Next, using Eqs. (D.30), (D.32) and (D.61) we find the coefficients $f_{2}(k)$ to be

$$
f_{2}(k)=f_{2}(0)+\sum_{m=1}^{k} \frac{B_{m}}{m^{2} R_{m}(M)},
$$

where

$$
B_{m}=\widehat{\Phi}_{2}(m)-\widehat{\Phi}_{2}(M)\left(1+R_{m}(M)\right) .
$$

Finally, taking the solution for $Q_{1}(u)$ from Eq. (5.30) with $\gamma_{0}=0$, we find the solution for $Q_{2}(u)$ in the form of Eq. (D.62) with

$$
B_{m}=\widehat{\Phi}_{2}(m)-\widehat{\Phi}_{2}(M)-\widetilde{b}(M) R_{m}(M) .
$$

\section{D.3.3 Another form for $Q_{1}(u)$}

As we explained earlier, the solution to the Baxter equation in the Mellin space is a sum of $z^{k}$ - and $(1-z)^{k}$-expansions (see Eq. (D.21)). However, the application of this form 
as the input to inhomogeneities on the right-hand side of the Baxter equation is not very convenient. It is a better choice to cast it solely in terms of the $z^{k}$-expansion,

$$
\Psi_{\ell}(z)=\sum_{k=0}^{M} z^{k} \widetilde{R}_{\ell, k}(M)
$$

For the four-loop calculation, considered in this study, the non-polynomial inhomogeneities contain only the functions $\Psi_{0}(z)$ and $\Psi_{1}(z)$. Moreover, the function $\Psi_{0}(z)$ and the $\widetilde{b}(M)$ as well as the $\gamma_{0}$ part of $\Psi_{1}(z)$ are symmetric under the interchange $z \leftrightarrow(1-z)$ and do not represent a difficulty on their own. Thus it is necessary to address the issue of non-symmetric contributions only for the remainder of the function $\Psi_{1}(z)$, with the coefficient $\sim R_{k}(M) S_{2}(k)$.

Our goal is to find a relation of the form

$$
\frac{1}{2} \sum_{k=0}^{M}\left[z^{k}+(1-z)^{k}\right] R_{k}(M) S_{2}(k)=\sum_{k=0}^{M} z^{k} \widetilde{R}_{2, k}(M)+K_{3} \Psi_{0}(z),
$$

where we also added the solution of the homogeneous Baxter equation with a coefficient $K_{3}$. To start with, we note that the left-hand side of Eq. (D.66) is a solution of the Baxter equation with the following right-hand side

$$
\frac{1}{2} \sum_{k=1}^{M}\left[z^{k-1}+(1-z)^{k-1}\right] R_{k}(M) .
$$

The second term here, i.e. $\sum_{k=0}^{M}(1-z)^{k-1} R_{k}(M)$, leads to the solution of the Baxter equation in the form $\sum_{k=0}^{M}(1-z)^{k} R_{k}(M) S_{2}(k)$. Thus, we merely have to replace the $(1-z)^{k}$-expansion by the $z^{k}$-expansion. Indeed, one can show, that

$$
\sum_{k=1}^{M}(1-z)^{k-1} R_{k}(M)=\sum_{k=1}^{M} z^{k-1} \sum_{m=1}^{k-1} R_{m}(M)
$$

and Eq. (D.67) can be represented as

$$
\sum_{k=1}^{M} z^{k-1} \sum_{m=1}^{k} R_{m}(M)
$$

Thus, the general solution to Eq. (D.67) admits the form

$$
\frac{1}{2} \sum_{k=0}^{M}\left[z^{k}+(1-z)^{k}\right] R_{k}(M) S_{2}(k)=\sum_{k=0}^{M} z^{k} R_{k}(M) V_{2,0}(k)+K_{3} \Psi_{0}(z),
$$

where $V_{2,0}(k)$ is given by Eq. (5.40) in the main text and the coefficient $K_{3}$ is fixed from numerical agreement between the left- and right-hand sides of Eq. (D.72)

$$
K_{3}=-2 S_{-2}(M) \text {. }
$$


The corresponding $u$-space form is then given by

$$
\sum_{k=0}^{M} \Re \mathrm{e}\left[P_{k}(u)\right] R_{k}(M) S_{2}(k)=\sum_{k=0}^{M} P_{k}(u) R_{k}(M) V_{2,0}(k)+K_{3} Q_{0}(u) .
$$

\section{D.4 Non-polynomial inhomogeneities}

Let us now address non-polynomial inhomogeneities emerging in the Baxter equation. To start with, we split the Baxter function (and its Mellin transform) at $\ell$-th order as

$$
Q_{\ell}(u+a i)=R_{\ell, 0}(M)+\widetilde{Q}_{\ell}(u+a i), \quad \Psi_{\ell}(z)=R_{\ell, 0}(M)+\widetilde{\Psi}_{\ell}(z),
$$

with $R_{0,0}(M)=1$, where

$$
\widetilde{Q}(u)=\sum_{k=1}^{M} \widehat{R}_{k}(M) P_{k}(u), \quad \widetilde{\Psi}(z)=K_{1} \sum_{k=1}^{M} \widehat{R}_{k}(M) z^{k},
$$

Then the inverse Mellin transform of a generic inhomogeneity is given by

$$
\begin{aligned}
\frac{L !}{\left(u^{+}\right)^{L+1}} \widetilde{Q}_{\ell}(u+i) & +(-1)^{L+1} \frac{L !}{\left(u^{-}\right)^{L+1}} \widetilde{Q}_{\ell}(u-i) \\
& \stackrel{M^{-1}}{\rightarrow}(-i)^{L+1} K \int_{0}^{\omega} \frac{d \omega_{1}}{\omega_{1}}\left(\ln \frac{\omega}{\omega_{1}}\right)^{L}\left(\frac{\omega}{\omega_{1}}\right)^{-b}\left\{-\omega_{1}^{\mp 1} \widetilde{Q}_{\ell}\left(-\omega_{1}\right)\right\} \\
& -i^{L+1} K \int_{\omega}^{\infty} \frac{d \omega_{1}}{\omega_{1}}\left(\ln \frac{\omega}{\omega_{1}}\right)^{L}\left(\frac{\omega}{\omega_{1}}\right)^{-b}\left\{-\omega_{1}^{\mp 1} \widetilde{Q}_{\ell}\left(-\omega_{1}\right)\right\} .
\end{aligned}
$$

After the substitution (D.14) for $\omega \rightarrow z$, we have for the r.h.s. of Eq. (D.75)

$$
\begin{aligned}
-(-i)^{L+1} K_{1} \sqrt{z(1-z)} & {\left[\frac{1}{z} \int_{0}^{z} \frac{d z_{1}}{z_{1}}\left(\ln \frac{z\left(1-z_{1}\right)}{z_{1}(1-z)}\right)^{L} \tilde{\Psi}_{s}\left(z_{1}\right)\right.} \\
+ & \left.\frac{1}{(1-z)} \int_{z}^{1} \frac{d z_{1}}{\left(1-z_{1}\right)}\left(\ln \frac{z_{1}(1-z)}{z\left(1-z_{1}\right)}\right)^{L} \tilde{\Psi}_{s}\left(z_{1}\right)\right] .
\end{aligned}
$$

The Baxter function $Q(u)$ and its Mellin transform $\Psi(z)$ possess symmetry properties preserved to all order of perturbation theory:

$$
Q(-u)=Q(u), \quad \Psi(1-z)=\Psi(z) .
$$

Thus, in all calculations we will focus on just one term in Eq. (D.76), say, the first one on the right-hand side, and then get the complete result for the Baxter function obeying the required symmetry properties (D.77) by adding contribution with reflected argument. With this argument in mind, Eqs. (D.75) and (D.76) can be rewritten as

$$
\frac{L !}{\left(u^{+}\right)^{L+1}} \widetilde{Q}_{\ell}(u+i)+(-1)^{L+1} \frac{L !}{\left(u^{-}\right)^{L+1}} \widetilde{Q}_{\ell}(u-i) \stackrel{M^{-1}}{\rightarrow}
$$




$$
-2(-i)^{L+1} K_{1} \frac{[z(1-z)]^{1 / 2}}{z} \int_{0}^{z} \frac{d z_{1}}{z_{1}}\left(\ln \frac{z\left(1-z_{1}\right)}{z_{1}(1-z)}\right)^{L} \widetilde{\Psi}_{\ell}\left(z_{1}\right) .
$$

To calculate the integral in the right-hand side of (D.78), it is convenient to re-express the integrand involving the logarithm as a rational function of its argument via

$$
\left(\ln \frac{z\left(1-z_{1}\right)}{z_{1}(1-z)}\right)^{L}=\left.\left(\frac{d}{d \varepsilon}\right)^{L}\left(\frac{z\left(1-z_{1}\right)}{z_{1}(1-z)}\right)^{\varepsilon}\right|_{\varepsilon=0} .
$$

Now, using the expansion for $\widetilde{\Psi}_{\ell}(z)$, after some algebra we obtain

$$
\int_{0}^{z} \frac{d z_{1}}{z_{1}}\left(\frac{z\left(1-z_{1}\right)}{z_{1}(1-z)}\right)^{\varepsilon} \widetilde{\Psi}_{\ell}\left(z_{1}\right)=\sum_{p=1}^{\infty} z^{p}\left[\frac{R_{p, \ell}(M)}{p-\varepsilon}+\frac{\varepsilon \Gamma(p)}{\Gamma(p+1-\varepsilon)} \sum_{k=1}^{p-1} R_{k, \ell}(M) \frac{\Gamma(k-\varepsilon)}{k !}\right] .
$$

The first term on the right-hand side of this equation is obviously polynomial, but the second one is not. Note, however, that for $p>M$ we can represent it as

$$
\begin{aligned}
\sum_{p=M+1}^{\infty} z^{p} \frac{\varepsilon \Gamma(p)}{\Gamma(p+1-\varepsilon)} & \sum_{k=1}^{p-1} R_{k, \ell}(M) \frac{\Gamma(k-\varepsilon)}{k !} \\
= & \sum_{p=M+1}^{\infty} z^{p} \frac{\varepsilon \Gamma(p)}{\Gamma(p+1-\varepsilon)} \sum_{k=1}^{M} R_{k, \ell}(M) \frac{\Gamma(k-\varepsilon)}{k !}
\end{aligned}
$$

where the inner sum in the last term can be cast in the form

$$
\sum_{k=1}^{M} R_{k, \ell}(M) \frac{\Gamma(k-\varepsilon)}{k ! \Gamma(-\varepsilon)}=\widetilde{Q}_{\ell}\left(u=\frac{i}{2}+i \varepsilon\right)=Q_{\ell}\left(u=\frac{i}{2}+i \varepsilon\right)-R_{0, s}(M) .
$$

Thus, we have

$$
\begin{aligned}
\int_{0}^{z} \frac{d z_{1}}{z_{1}}\left(\frac{z\left(1-z_{1}\right)}{z_{1}(1-z)}\right)^{\varepsilon} \tilde{\Psi}_{\ell}\left(z_{1}\right) & =\sum_{p=1}^{M} z^{p}\left[\frac{R_{p, \ell}(M)}{p-\varepsilon}+\frac{\varepsilon \Gamma(p)}{\Gamma(p+1-\varepsilon)} \sum_{k=1}^{p-1} R_{k, \ell}(M) \frac{\Gamma(k-\varepsilon)}{k !}\right] \\
& -\sum_{p=M+1}^{\infty} z^{p} \frac{\Gamma(p) \Gamma(1-\varepsilon)}{\Gamma(p+1-\varepsilon)} \widetilde{Q}_{\ell}\left(u=\frac{i}{2}+i \varepsilon\right) .
\end{aligned}
$$

To get back to the integrand in question, we have to differentiate both sides w.r.t. $\varepsilon$ and set it to zero. Then one immediately finds that the last term becomes

$$
-\left.\sum_{k=0}^{L} C_{L}^{k} \sum_{p=M+1}^{\infty} \frac{z^{p}}{p} i^{k} \widetilde{Q}_{\ell}^{(k)}\left(\frac{i}{2}\right)\left(\frac{d}{d \varepsilon}\right)^{L-k}\left\{\frac{\Gamma(p) \Gamma(1-\varepsilon)}{\Gamma(p+1-\varepsilon)}\right\}\right|_{\varepsilon=0},
$$

where we introduced notations for

$$
C_{L}^{k}=\frac{L !}{k !(L-k) !}, \quad \widetilde{Q}_{\ell}^{(k)}\left( \pm \frac{i}{2}\right)=\left(\frac{d}{d u}\right)^{k} \widetilde{Q}_{\ell}\left(u= \pm \frac{i}{2}\right), \quad \widetilde{Q}_{\ell}^{(0)}\left( \pm \frac{i}{2}\right)=\widetilde{Q}_{\ell}\left( \pm \frac{i}{2}\right)
$$


and for the coefficients

$$
Z_{L}(p, k)=\left.\left(\frac{d}{d \varepsilon}\right)^{L}\left\{\frac{\Gamma(k+1-\varepsilon)}{\Gamma(p+1-\varepsilon)}\right\}\right|_{\varepsilon=0},
$$

which are expressed in terms of harmonic numbers as follows for a few low values of $L$,

$$
\begin{aligned}
Z_{0}(p, k) & =1 \\
Z_{1}(p, k) & =S_{1}(p, k), \\
Z_{2}(p, k) & =S_{1}^{2}(p, k)+S_{2}(p, k), \\
Z_{3}(p, k) & =S_{1}^{3}(p, k)+3 S_{1}(p, k) S_{2}(p, k)+2 S_{3}(p, k), \\
Z_{4}(p, k) & =S_{1}^{4}(p, k)+6 S_{1}^{2}(p, k) S_{2}(p, k)+8 S_{1}(p, k) S_{3}(p, k)+3 S_{2}^{2}(p, k)+6 S_{4}(p, k), \\
Z_{5}(p, k) & =S_{1}^{5}(p, k)+10 S_{1}^{3}(p, k) S_{2}(p, k)+20 S_{1}^{2}(p, k) S_{3}(p, k)+15 S_{1}(p, k) S_{2}^{2}(p, k) \\
& +30 S_{1}(p, k) S_{4}(p, k)+20 S_{2}(p, k) S_{3}(p, k)+24 S_{5}(p, k),
\end{aligned}
$$

where

$$
S_{L}(p, k)=S_{L}(p)-S_{L}(k)
$$

and $Z_{L}(p)=Z_{L}(p, 0)$.

Assembling all results, we have

$$
\begin{gathered}
\frac{1}{\left(u^{+}\right)^{L+1}} \widetilde{Q}_{\ell}(u+i)+\frac{(-1)^{L+1}}{\left(u^{-}\right)^{L+1}} \widetilde{Q}_{\ell}(u-i) \stackrel{M^{-1}}{\rightarrow} \\
-2(-i)^{L+1} K_{1} \sqrt{z(1-z)}\left[\sum_{p=1}^{M} \frac{z^{p-1}}{p}\left\{\frac{R_{p, \ell}(M)}{p^{L}}+\frac{1}{(L-1) !} \sum_{k=1}^{p-1} \frac{R_{k, \ell}(M)}{k} Z_{L-1}(p, k-1)\right\}\right. \\
\left.-\sum_{k=0}^{L} \frac{i^{l}}{k !(L-k) !} \widetilde{Q}_{\ell}^{(k)}\left(\frac{i}{2}\right) \sum_{p=M+1}^{\infty} \frac{z^{p-1}}{p} Z_{L-k}(p)\right] .
\end{gathered}
$$

And after returning to the $u$-space the final result, free from Stirling numbers, reads

$$
\begin{aligned}
& \frac{1}{\left(u^{+}\right)^{L+1}} \widetilde{Q}_{\ell}(u+i)+\frac{(-1)^{L+1}}{\left(u^{-}\right)^{L+1}} \widetilde{Q}_{\ell}(u-i) \\
&=-2(-i)^{L+1} {\left[\sum_{p=1}^{M} \frac{P_{p-1}(u)}{p}\left\{\frac{R_{p, \ell}(M)}{p^{L}}+\frac{1}{(L-1) !} \sum_{k=1}^{p-1} \frac{R_{k, \ell}(M)}{k} Z_{L-1}(p, k-1)\right\}\right.} \\
&\left.-\sum_{k=0}^{L} \frac{i^{k}}{k !(L-k) !} \widetilde{Q}_{\ell}^{(k)}\left(\frac{i}{2}\right) \sum_{p=M+1}^{\infty} \frac{P_{p-1}(u)}{p} Z_{L-k}(p)\right]
\end{aligned}
$$

where $P_{p-1}(u)$ is given in Eq. (5.3). Note that for $L>0$, Eq. (D.90) can be simplified to

$$
\frac{1}{\left(u^{+}\right)^{L+1}} \widetilde{Q}_{\ell}(u+i)+\frac{(-1)^{L+1}}{\left(u^{-}\right)^{L+1}} \widetilde{Q}_{\ell}(u-i)
$$




$$
\begin{aligned}
=-2(-i)^{L+1} & {\left[\frac{1}{(L-1) !} \sum_{p=1}^{M} \frac{P_{p-1}(u)}{p} \sum_{k=1}^{p} \frac{R_{k, \ell}(M)}{k} Z_{L-1}(p, k-1)\right.} \\
& \left.-\sum_{k=0}^{L} \frac{i^{k}}{k !(L-k) !} \widetilde{Q}_{\ell}^{(k)}\left(\frac{i}{2}\right) \sum_{p=M+1}^{\infty} \frac{P_{p-1}(u)}{p} Z_{L-k}(p)\right] .
\end{aligned}
$$

Repeating all above calculations, it is possible to obtain in an analogous fashion

$$
\begin{aligned}
& \frac{1}{\left(u^{+}\right)^{L+1}} \widetilde{Q}_{\ell}(u)+\frac{(-1)^{L+1}}{\left(u^{-}\right)^{L+1}} \widetilde{Q}_{\ell}(u) \\
& \stackrel{M^{-1}}{\rightarrow} 2(-i)^{L+1} K_{1} \sqrt{z(1-z)}\left[\frac{1}{L !} \sum_{p=1}^{M} \frac{z^{p-1}}{p} \sum_{k=1}^{p-1} \frac{R_{k, \ell}(M)}{k} Z_{L}(p, k)\right. \\
& \left.\quad+\sum_{k=0}^{L} \frac{i^{k}}{k !(L-k) !} \widetilde{Q}_{\ell}^{(k)}\left(-\frac{i}{2}\right) \sum_{p=M+1}^{\infty} \frac{z^{p-1}}{p} Z_{L-k}(p)\right],
\end{aligned}
$$

and as a consequence

$$
\begin{aligned}
\frac{1}{\left(u^{+}\right)^{L+1}} \widetilde{Q}_{\ell}(u)+ & \frac{(-1)^{L+1}}{\left(u^{-}\right)^{L+1}} \widetilde{Q}_{\ell}(u) \\
= & 2(-i)^{L+1}\left[\frac{1}{L !} \sum_{p=1}^{M} \frac{P_{p-1}(u)}{p} \sum_{k=1}^{p-1} R_{k, \ell}(M) Z_{L}(p, k)\right. \\
& \left.+\sum_{k=0}^{L} \frac{i^{k}}{k !(L-k) !} \widetilde{Q}_{\ell}^{(k)}\left(-\frac{i}{2}\right) \sum_{p=M+1}^{\infty} \frac{P_{p-1}(u)}{p} Z_{L-k}(p)\right] .
\end{aligned}
$$

Last but not least, as a particular case of (D.92) and (D.93), we find

$$
\begin{aligned}
& \frac{1}{\left(u^{+}\right)^{L+1}}+\frac{(-1)^{L+1}}{\left(u^{-}\right)^{L+1}} \stackrel{M^{-1}}{\rightarrow} 2(-i)^{L+1} K_{1} \sqrt{z(1-z)} \sum_{p=1}^{\infty} \frac{z^{p-1}}{p} Z_{L}(p), \\
& \frac{1}{\left(u^{+}\right)^{L+1}}+\frac{(-1)^{L+1}}{\left(u^{-}\right)^{L+1}}=2(-i)^{L+1} \sum_{p=1}^{\infty} \frac{P_{p-1}(u)}{p} Z_{L}(p) .
\end{aligned}
$$

\section{E On the degree reducing constants}

We have seen that the polynomial-type contribution to the Baxter functions takes the form of a linear combination of higher derivatives $T_{(a, b, c, \ldots)}$ plus a constant times the leading order Baxter polynomial $Q_{0}$. This constant is fixed by the requirement that the monomial $u^{M}$ cancels in the total contribution. Thus, the following ratios are all we need to determine the degree reducing constant

$$
\rho_{(a, b, c, \ldots)}=\frac{\text { coefficient of } u^{M} \text { in } T_{(a, b, c, \ldots)}}{\text { coefficient of } u^{M} \text { in } Q_{0}} .
$$


Here, we list the cases which are needed for computing $a_{2}$, and $a_{3}$. The notation is

$$
\begin{aligned}
S_{a, b, \ldots}= & S_{a, b, \ldots}(M), \quad \widetilde{S}_{a, b, \ldots}=S_{a, b, \ldots}(2 M), \quad \widehat{S}_{a, b, \ldots}=S_{a, b, \ldots}(M / 2) \\
\rho_{(1,0)}= & 2 \widetilde{S}_{1}-3 S_{1}, \\
\rho_{(0,1)}= & 2 \widehat{S}_{2}, \\
\rho_{(2,0)}= & 9 S_{1}^{2}-12 \widetilde{S}_{1} S_{1}+4 \widetilde{S}_{1}^{2}+5 S_{2}-4 \widetilde{S}_{2}, \\
\rho_{(1,1)}= & -6 S_{1} \widehat{S}_{2}+4 \widetilde{S}_{1} \widehat{S}_{2}-2 \widehat{S}_{3}, \\
\rho_{(0,2)}= & 12 \widehat{S}_{2}^{2}+12 \widehat{S}_{4} \\
\rho_{(3,0)}= & -27 S_{1}^{3}+54 \widetilde{S}_{1} S_{1}^{2}-36 \widetilde{S}_{1}^{2} S_{1}-45 S_{2} S_{1}+36 \widetilde{S}_{2} S_{1}+ \\
& +8 \widetilde{S}_{1}^{3}-18 S_{3}+30 S_{2} \widetilde{S}_{1}-24 \widetilde{S}_{1} \widetilde{S}_{2}+16 \widetilde{S}_{3}, \\
\rho_{(2,1)}= & 18 \widehat{S}_{2} S_{1}^{2}+12 \widehat{S}_{3} S_{1}-24 \widehat{S}_{2} \widetilde{S}_{1} S_{1}+2 \widehat{S}_{2}^{2}+8 \widehat{S}_{2} \widetilde{S}_{1}^{2}+ \\
& +10 S_{2} \widehat{S}_{2}+6 \widehat{S}_{4}-8 \widehat{S}_{3} \widetilde{S}_{1}-8 \widehat{S}_{2} \widetilde{S}_{2}, \\
\rho_{(1,2)}= & -36 S_{1} \widehat{S}_{2}^{2}+24 \widetilde{S}_{1} \widehat{S}_{2}^{2}-24 \widehat{S}_{3} \widehat{S}_{2}-36 \widehat{S}_{1} \widehat{S}_{4}-24 \widehat{S}_{5}+24 \widehat{S}_{4} \widetilde{S}_{1}, \\
\rho_{(0,3)}= & 120 \widehat{S}_{2}^{3}+360 \widehat{S}_{4} \widehat{S}_{2}+240 \widehat{S}_{6} .
\end{aligned}
$$

In the case of $b_{3}$, we need the ratios

$$
\begin{aligned}
\rho_{(0,0,2)}= & 2 S_{2}, \\
\rho_{(0,0,4)}= & 12 S_{2}^{2}+12 S_{4}, \\
\rho_{(0,0,4)}= & 12 S_{2}^{2}+12 S_{4}, \\
\rho_{(0,0,4)}= & 12 S_{2}^{2}+12 S_{4}, \\
\rho_{(0,0,6)}= & 120 S_{2}^{3}+360 S_{4} S_{2}+240 S_{6}, \\
\rho_{(0,2,0)}= & 4 S_{1}^{2}+2 S_{2}, \\
\rho_{(0,2,2)}= & 8 S_{2} S_{1}^{2}+16 S_{3} S_{1}+4 S_{2}^{2}+12 S_{4}, \\
\rho_{(0,2,2)}= & 8 S_{2} S_{1}^{2}+16 S_{3} S_{1}+4 S_{2}^{2}+12 S_{4}, \\
\rho_{(0,2,4)}= & 24 S_{2}^{3}+48 S_{1}^{2} S_{2}^{2}+192 S_{1} S_{3} S_{2}+168 S_{4} S_{2}+96 S_{3}^{2}+48 S_{1}^{2} S_{4}+ \\
& +192 S_{1} S_{5}+240 S_{6}, \\
\rho_{(0,3,0)}= & -8 S_{1}^{3}-12 S_{2} S_{1}-4 S_{3}, \\
\rho_{(0,3,2)}= & -16 S_{2} S_{1}^{3}-48 S_{3} S_{1}^{2}-24 S_{2}^{2} S_{1}-72 S_{4} S_{1}-32 S_{2} S_{3}-48 S_{5}, \\
\rho_{(0,4,0)}= & 16 S_{1}^{4}+48 S_{2} S_{1}^{2}+32 S_{3} S_{1}+12 S_{2}^{2}+12 S_{4}, \\
\rho_{(0,4,0)}= & 16 S_{1}^{4}+48 S_{2} S_{1}^{2}+32 S_{3} S_{1}+12 S_{2}^{2}+12 S_{4}, \\
\rho_{(0,4,0)}= & 16 S_{1}^{4}+48 S_{2} S_{1}^{2}+32 S_{3} S_{1}+12 S_{2}^{2}+12 S_{4}, \\
\rho_{(0,4,2)}= & 32 S_{2} S_{1}^{4}+128 S_{3} S_{1}^{3}+96 S_{2}^{2} S_{1}^{2}+288 S_{4} S_{1}^{2}+256 S_{2} S_{3} S_{1}+ \\
& +384 S_{5} S_{1}+24 S_{2}^{3}+64 S_{3}^{2}+168 S_{2} S_{4}+240 S_{6}, \\
\rho_{(0,5,0)}= & -32 S_{1}^{5}-160 S_{2} S_{1}^{3}-160 S_{3} S_{1}^{2}-120 S_{2}^{2} S_{1}-120 S_{4} S_{1}-80 S_{2} S_{3}-48 S_{5},
\end{aligned}
$$




$$
\begin{aligned}
\rho_{(0,6,0)}= & 64 S_{1}^{6}+480 S_{2} S_{1}^{4}+640 S_{3} S_{1}^{3}+720 S_{2}^{2} S_{1}^{2}+720 S_{4} S_{1}^{2}+960 S_{2} S_{3} S_{1}+ \\
& +576 S_{5} S_{1}+120 S_{2}^{3}+160 S_{3}^{2}+360 S_{2} S_{4}+240 S_{6}, \\
\rho_{(1,0,0)}= & \tilde{S}_{1}-2 S_{1} \\
\rho_{(1,0,2)}= & -4 S_{1} S_{2}+2 \tilde{S}_{1} S_{2}-2 S_{3}, \\
\rho_{(1,0,4)}= & -24 S_{1} S_{2}^{2}+12 \tilde{S}_{1} S_{2}^{2}-24 S_{3} S_{2}-24 S_{1} S_{4}-24 S_{5}+12 S_{4} \tilde{S}_{1}, \\
\rho_{(1,2,0)}= & -8 S_{1}^{3}+4 \tilde{S}_{1} S_{1}^{2}-8 S_{2} S_{1}-2 S_{3}+2 S_{2} \tilde{S}_{1} \\
\rho_{(1,2,2)}= & -16 S_{2} S_{1}^{3}-40 S_{3} S_{1}^{2}+8 S_{2} \tilde{S}_{1} S_{1}^{2}-16 S_{2}^{2} S_{1}-48 S_{4} S_{1}+16 S_{3} \tilde{S}_{1} S_{1}+ \\
& -16 S_{2} S_{3}-24 S_{5}+4 S_{2}^{2} \tilde{S}_{1}+12 S_{4} \tilde{S}_{1} \\
\rho_{(1,3,0)}= & 16 S_{1}^{4}-8 \tilde{S}_{1} S_{1}^{3}+36 S_{2} S_{1}^{2}+20 S_{3} S_{1}-12 S_{2} \tilde{S}_{1} S_{1}+6 S_{2}^{2}+6 S_{4}-4 S_{3} \tilde{S}_{1}, \\
\rho_{(1,4,0)}= & -32 S_{1}^{5}+16 \tilde{S}_{1} S_{1}^{4}-128 S_{2} S_{1}^{3}-112 S_{3} S_{1}^{2}+48 S_{2} \tilde{S}_{1} S_{1}^{2}-72 S_{2}^{2} S_{1}+ \\
& -72 S_{4} S_{1}+32 S_{3} \tilde{S}_{1} S_{1}-40 S_{2} S_{3}-24 S_{5}+12 S_{2}^{2} \tilde{S}_{1}+12 S_{4} \tilde{S}_{1}, \\
\rho_{(2,0,0)}= & 4 S_{1}^{2}-4 \tilde{S}_{1} S_{1}+\tilde{S}_{1}^{2}+2 S_{2}-\tilde{S}_{2}, \\
\rho_{(2,0,2)}= & 8 S_{2} S_{1}^{2}+8 S_{3} S_{1}-8 S_{2} \tilde{S}_{1} S_{1}+6 S_{2}^{2}+2 S_{2} \tilde{S}_{1}^{2}+6 S_{4}-4 S_{3} \tilde{S}_{1}-2 S_{2} \tilde{S}_{2}, \\
\rho_{(2,2,0)}= & 16 S_{1}^{4}-16 \tilde{S}_{1} S_{1}^{3}+4 \tilde{S}_{1}^{2} S_{1}^{2}+32 S_{2} S_{1}^{2}-4 \tilde{S}_{2} S_{1}^{2}+16 S_{3} S_{1}+ \\
& -16 S_{2} \tilde{S}_{1} S_{1}+6 S_{2}^{2}+2 S_{2} \tilde{S}_{1}^{2}+6 S_{4}-4 S_{3} \tilde{S}_{1}-2 S_{2} \tilde{S}_{2}, \\
\rho_{(3,0,0)}= & -8 S_{1}^{3}+12 \tilde{S}_{1} S_{1}^{2}-6 \tilde{S}_{1}^{2} S_{1}-12 S_{2} S_{1}+6 \tilde{S}_{2} S_{1}+\tilde{S}_{1}^{3}+ \\
& -4 S_{3}+6 S_{2} \tilde{S}_{1}-3 \tilde{S}_{1} \tilde{S}_{2}+2 \tilde{S}_{3} .
\end{aligned}
$$

\section{References}

[1] J.A.M. Vermaseren, S. Moch, Mathematics for structure functions, Nucl. Phys. Proc. Suppl. 89 (2000) 131.

[2] V.M. Braun, S.E. Derkachov, A.N. Manashov, Phys. Rev. Lett. 81 (1998) 2020; V.M. Braun, S.E. Derkachov, G.P. Korchemsky, A.N. Manashov, Nucl. Phys. B 553 (1999) 355.

[3] A.V. Belitsky, Phys. Lett. B 453 (1999) 59; Nucl. Phys. B 574 (2000) 407.

[4] L.N. Lipatov, Evolution equations in QCD, in Perspectives in Hadronic Physics, eds. S. Boffi, C. Ciofi Degli Atti, M. Giannini, World Scientific (Singapore, 1998) p. 413.

[5] N. Beisert, M. Staudacher, Nucl. Phys. B 727 (2005) 1.

[6] A.V. Belitsky, S.E. Derkachov, G.P. Korchemsky, A.N. Manashov, Phys. Lett. B 594 (2004) 385; Nucl. Phys. B 708 (2005) 115.

[7] J.M. Maldacena, Adv. Theor. Math. Phys. 2 (1998) 231;

S.S. Gubser, I.R. Klebanov, A.M. Polyakov, Phys. Lett. B 428 (1998) 105;

E. Witten, Adv. Theor. Math. Phys. 2 (1998) 253. 
[8] I. Bena, J. Polchinski, R. Roiban, Phys. Rev. D 69 (2004) 046002.

[9] N. Beisert, B. Eden, M. Staudacher, J. Stat. Mech. 0701 (2007) P021.

[10] Z. Bern, M. Czakon, L.J. Dixon, D.A. Kosower, V.A. Smirnov, Phys. Rev. D 75 (2007) 085010;

F. Cachazo, M. Spradlin, A. Volovich, Phys. Rev. D 75 (2007) 105011.

[11] R. Roiban and A. A. Tseytlin, JHEP 0711 (2007) 016;

B. Basso, G. P. Korchemsky and J. Kotanski, Phys. Rev. Lett. 100 (2008) 091601.

[12] M. Beccaria, V. Forini, A. Tirziu and A. A. Tseytlin, Nucl. Phys. B 812 (2009) 144;

L. Freyhult and S. Zieme, Phys. Rev. D 79, 105009 (2009).

[13] A.V. Belitsky, Phys. Lett. B 643 (2006) 354.

[14] A.V. Belitsky, Phys. Lett. B 677 (2009) 93.

[15] A.V. Kotikov, L.N. Lipatov, V.N. Velizhanin, Phys. Lett. B 557 (2003) 114

[16] S. Moch, J.A.M. Vermaseren, A. Vogt, Nucl. Phys. B 688 (2004) 101.

[17] A.V. Kotikov, L.N. Lipatov, A.I. Onishchenko, V.N. Velizhanin, Phys. Lett. B 595 (2004) 521; (E) Phys. Lett. B 632 (2006) 754.

[18] F. Fiamberti, A. Santambrogio, C. Sieg, D. Zanon, Phys. Lett. B 666 (2008) 100.

[19] V.N. Velizhanin, The Four-Loop Konishi in N = 4 SYM, arXiv:0808.3832 [hep-th]; Leading transcedentality contributions to the four-loop universal anomalous dimension in $N=4 S Y M$, arXiv:0811.0607 [hep-th].

[20] A.V. Kotikov, L.N. Lipatov, Nucl. Phys. B 661 (2003) 19 [Erratum-ibid. B 685 (2004) 405].

[21] Z. Bajnok, R.A. Janik, T. Lukowski, Nucl. Phys. B 816 (2009) 376.

[22] M. Beccaria, V. Forini, T. Lukowski, S. Zieme, J. High Ener. Phys. 0903 (2009) 129.

[23] A.V. Kotikov, A. Rej, S. Zieme, Nucl. Phys. B 813 (2009) 460.

[24] J. Ambjorn, R.A. Janik, C. Kristjansen, Nucl. Phys. B 736 (2006) 288.

[25] A.V. Kotikov, L.N. Lipatov, A. Rej, M. Staudacher, V.N. Velizhanin, J. Stat. Mech. 0710 (2007) P10003.

[26] N. Beisert, V. Dippel, M. Staudacher, J. High Ener. Phys. 0407 (2004) 075.

[27] A.V. Kotikov, V.N. Velizhanin, Analytic continuation of the Mellin moments of deep inelastic structure functions, arXiv:hep-ph/0501274. 
[28] L.D. Faddeev, G.P. Korchemsky, Phys. Lett. B 342 (1995) 311.

[29] Yu.L. Dokshitzer, G. Marchesini, Phys. Lett. B 646 (2007) 189.

[30] B. Basso, G.P. Korchemsky, Nucl. Phys. B 775 (2007) 1.

[31] M. Beccaria, V. Forini, J. High. Ener. Phys. 0903 (2009) 111.

[32] V. Forini, M. Beccaria, Theor. Math. Phys. 159 (2009) 712.

[33] R. Askey, J. Wilson, Some basic hypergeometric orthogonal polynomials that generalize Jacobi polynomials, Mem. Am. Math. Soc. 54 (1985) 319. 